Revue internationale P.M.E.

\title{
Les leviers sociaux de la réussite entrepreneuriale
} essai de validation empirique dans le secteur des TIC

\section{The social levers of the entrepreneurial success}

An attempt of an empirical validation in the Information

Technology field

\section{Las medidas sociales del éxito empresarial enseyo de validación empírica en el sector del TIC}

\section{Amina Omrane et Olfa Zeribi-Ben Slimane}

Volume 27, numéro 3-4, 2014

URI : https://id.erudit.org/iderudit/1028041ar

DOI : https://doi.org/10.7202/1028041ar

Aller au sommaire du numéro

\section{Éditeur(s)}

Editions EMS - In Quarto SARL

\section{ISSN}

0776-5436 (imprimé)

1918-9699 (numérique)

Découvrir la revue

\section{Citer cet article}

Omrane, A. \& Zeribi-Ben Slimane, O. (2014). Les leviers sociaux de la réussite entrepreneuriale : essai de validation empirique dans le secteur des TIC. Revue internationale P.M.E., 27(3-4), 71-103. https://doi.org/10.7202/1028041ar

\section{Résumé de l'article}

Le capital social constitue un outil pertinent non seulement pour développer le réseau de relations sociales que détient l'entrepreneur avec les parties prenantes clés au développement de son entreprise nouvellement créée, mais également pour favoriser son accès aux ressources externes stratégiques, à savoir le financement et les informations requises.

Or, la rareté des recherches consacrées aux antécédents du capital social entrepreneurial nous a interpellées et amenées à tenter d'approfondir notre appréhension du processus par lequel l'entrepreneur pourrait contribuer à faire perdurer son entreprise nouvelle. Il serait donc judicieux de porter une attention particulière aux facteurs qui sont susceptibles de faciliter la formation et le développement du capital social entrepreneurial.

Une étude menée auprès de 120 entrepreneurs de sociétés de services et d'ingénierie informatique (SSII) tunisiennes illustre le rôle que jouent les compétences sociales de l'entrepreneur dans le développement d'un capital social (découlant d'un réseau étendu, de relations riches en liens faibles et non redondants) propice à un accès plus facile aux informations et au financement requis. Ces compétences sociales renferment le management de l'impression via la valorisation d'autrui, la persuasion sociale et l'intelligence émotionnelle de soi.
Tous droits réservés @ Editions EMS - In Quarto SARL, 2014

Ce document est protégé par la loi sur le droit d'auteur. L'utilisation des services d'Érudit (y compris la reproduction) est assujettie à sa politique d'utilisation que vous pouvez consulter en ligne.

https://apropos.erudit.org/fr/usagers/politique-dutilisation/ 


\title{
Les leviers sociaux de la réussite entrepreneuriale : essai de validation empirique dans le secteur des TIC
}

\begin{abstract}
Amina OMRANE
Amina Omrane est docteure et maître-assistante en sciences de gestion et entrepreneuriat à l'Université de Sfax (Tunisie). Elle a préparé sa thèse de doctorat en cotutelle entre l'Université Jean Moulin de Lyon 3 et l'IHEC de Carthage. Elle enseigne des matières reliées à la politique et stratégie d'entreprise, à la culture entrepreneuriale et au management. Elle est également chercheure-associée en sciences de gestion, membre du laboratoire de recherche ECSTRA à l'IHEC (Carthage) et du centre de recherche en entrepreneuriat à l'EM Lyon Business School (Lyon).
\end{abstract}

Route de Tunis Km 5-5

3021 SAKIET EZZIT-SFAX, Tunisie

amina.omrane@yahoo.fr

\section{Olfa ZERIBI-BEN SLIMANE}

Olfa Zeribi-Ben Slimane, est diplômée de l'Institut d'administration des entreprises et de l'Institut de gestion de Rennes, est professeure en sciences de gestion à l'Institut des hautes études commerciales à Carthage. Expert auprès du programme des Nations unies pour le développement, comme consultante auprès d'organismes privés/publics. Formatrice au sein de l'Institut de développement des compétences des hauts fonctionnaires d'État. Ses champs de compétences et publications portent sur la responsabilité sociétale des entreprises - le management et la stratégie - le management et la gouvernance publique.

IHEC Carthage - Carthage Présidence
2016 TUNIS, Tunisie
zeribi.olfa@gmail.com

\section{RÉSUMÉ}

Le capital social constitue un outil pertinent non seulement pour développer le réseau de relations sociales que détient l'entrepreneur avec les parties prenantes clés au développement de son entreprise nouvellement créée, mais également pour favoriser son accès aux ressources externes stratégiques, à savoir le financement et les informations requises.

Or, la rareté des recherches consacrées aux antécédents du capital social entrepreneurial nous a interpellées et amenées à tenter d'approfondir notre appréhension du processus par lequel l'entrepreneur pourrait contribuer à faire perdurer son entreprise nouvelle. Il serait donc judicieux de porter une attention particulière aux facteurs qui sont susceptibles de faciliter la formation et le développement $d u$ capital social entrepreneurial.

Une étude menée auprès de 120 entrepreneurs de sociétés de services et d'ingénierie informatique (SSII) tunisiennes illustre le rôle que jouent les compétences sociales de l'entrepreneur dans le développement 
d'un capital social (découlant d'un réseau étendu, de relations riches en liens faibles et non redondants) propice à un accès plus facile aux informations et au financement requis. Ces compétences sociales renferment le management de l'impression via la valorisation d'autrui, la persuasion sociale et l'intelligence émotionnelle de soi.

MOTS CLÉS

Capital social de l'entrepreneur. Compétences sociales, Accès aux ressources externes clés

\title{
The social levers of the entrepreneurial success. An attempt of an empirical validation in the Information Technology field
}

\begin{abstract}
The social capital constitutes a relevant tool for developing the network of social relations that the entrepreneur entertains with the stakeholders who are essential for the development of his new venture. It is also so important for facilitating his access to external strategic resources, namely financing and useful information.

However, the scarcity of researches devoted to the antecedents of the entrepreneurial social capital brought us to try to deepen our apprehension of the process by which the entrepreneur could contribute to sustain his new company. Accordingly, it was more compelling to pay a special attention to the factors that are more likely to facilitate the formation and the development of the entrepreneurial social capital. A survey undertaken on 120 Information Technology (IT) Tunisian entrepreneurs illustrate the role that social skills play in the development of an entrepreneurial social capital (rising from a large network rich in weak and non redundant ties) which is favorable for accessing easily to the necessary information and financing.

These social skills encompass management impression-via the valorization of others, social persuasion and personal emotional intelligence.
\end{abstract}

KEYWORDS

Entrepreneurial social capital, Social skills, Access to external key resources

\section{Las medidas sociales del éxito empresarial : enseyo de validación empirica en el sector del TIC}

\begin{abstract}
RESUMEN
El capital social permite el desarrollo de la red de relaciones sociales del empresario con las partes involucradas en el desarrollo de su nueva empresa. También, es relevante para ayudarle a acceder a varios recursos externos estratégicos como la financiación y las informaciones.

Sin embargo, fuimos interpeladas por la rareza de las investigaciones sobre los antecedentes del capital social empresarial. Eso nos condujo a intentar profundizar nuestra comprensión del proceso que permite al empedrador hacer durar su nueva empresa. Por lo tanto, sería conveniente de interesarse en los factores susceptibles de ayudar a la formación y al desarrollo del capital social empresarial.

El estudio de 120 dirigentes de empresas de servicios de ingeniería informática muestra el papel jugado por las competencias sociales del dirigente en el desarrollo de un capital social (resultante de un red
\end{abstract}


amplio, de relaciones ricas en vínculos débiles y sin redundancia) que permite un acceso más fácil a las informaciones y a la financiación requerida. Estas competencias sociales reagrupan la gestión de la impresión por la valorización del otro, la persuasión social y la inteligencia emocional de sí.

Palabras Claves

Capital social del empresario, Competencias sociales, Acceso a los recursos externos

\section{INTRODUCTION}

Nombreuses sont les recherches qui ont souligné l'importance que revêt la notion de ressources tout au long du processus de création d'entreprises, et plus spécifiquement lors de la phase de survie-développement des entreprises nouvellement créées (Cooper, Folta et Woo, 1991 ; Chandler et Hanks, 1994 ; Carter, Williams et Reynolds, 1997 ; Green et Brown, 1997). Carter, Williams et Reynolds (p. 128) ont conforté ces assomptions de la sorte : " a firm's resources at start-up are critical determinants of success and survival ».

En effet, les entreprises nouvellement créées souffrent, le plus souvent, d'un manque de légitimité dû au handicap de la nouveauté "the liability of newness ", de l'incertitude, ainsi que de la rareté des ressources disponibles. Le secteur des TIC n'échappe pas à cette règle. Le nombre élevé d'entrées/sorties en TIC, ainsi que les problèmes rencontrés par plusieurs sociétés de services et d'ingénierie informatique créées, notamment au-delà de leur troisième année d'existence, mettent en perspective les risques qui peuvent accompagner la mise en place et la croissance de tels projets.

Ce scepticisme remettant en cause la réussite des nouvelles entreprises en TIC nous a interpellées et permis de relancer le débat autour des facteurs qui contribuent au succès entrepreneurial via la recherche, la sélection et l'acquisition des ressources clés. Ces ressources étant diverses et variées, nous avons retenu celles qui ont été, de notre point de vue, les plus évoquées au niveau des travaux récemment menés dans le champ de l'entrepreneuriat, à savoir : les ressources financières et informationnelles. Mais, quel est le mode d'accès aux ressources le plus privilégié pour les entrepreneurs qui sont en processus de concrétisation de leurs projets entrepreneuriaux?

Une lignée de recherches s'est développée afin de mettre en évidence le rôle moteur que joue le capital social de l'entrepreneur dans son accès aux ressources externes (Nahapiet et Ghoshal, 1998 ; Davidsson et Honig, 2003 ; Florin, Lubatkin et Schulze, 2003 ; Uzzi, 1997, 1999; Shane et Cable, 2002 ; Aldrich et Ruef, 2006). Or, les études ont largement mis en perspective le capital social entrepreneurial et ses retombées positives sur la réussite des nouveaux projets de création, au point de l'assimiler à la facilitation du processus entrepreneurial (Anderson et Jack, 2002 ; Julien, 2005). Cependant, en dépit de l'intérêt porté aux effets du capital social, la question de ses antécédents a été négligée. En effet, nous dénombrons peu de travaux qui ont essayé d'apporter un éclairage sur les déterminants de la formation et du développement du capital social. Dans ce sens, Ventolini (2010) souligne que ce capital n'est autre que le produit de l'insertion de l'entrepreneur dans des réseaux sociaux pour lui procurer les ressources nécessaires au développement de son projet ; les facteurs susceptibles de contribuer à sa constitution semblent être faiblement abordés par la littérature. À ce stade, nous nous sommes demandé si les compétences sociales de l'entrepreneur TIC jouent un rôle. 
Ce questionnement incombe du fait que quelques recherches conceptuelles, réalisées essentiellement par Markman et Baron (1998), Baron et Markman (2000, 2003), Baron (2004a, b), ainsi que Baron et Tang (2009), ont démontré que les compétences sociales de l'entrepreneur contribuent à faciliter son accès aux ressources externes, d'autant plus qu'elles impactent positivement l'extension de son capital social. De tels propos feront l'objet d'un approfondissement ultérieur.

Ainsi, cette recherche cherche à répondre au questionnement suivant : quel est le rôle que jouent les compétences sociales de l'entrepreneur TIC dans le développement de son capital social afin de favoriser son accès aux ressources externes requises au développement de son projet entrepreneurial ? Cette recherche vise ainsi à démontrer que les compétences sociales de l'entrepreneur lui ouvrent la voie vers l'exploitation d'un capital social propice à lui procurer les ressources sollicitées pour la maturation de son entreprise nouvelle.

Le contenu de cette recherche sera organisé comme suit : dans un premier temps, nous examinerons le cadrage théorique en approfondissant notre compréhension des concepts de compétences sociales, de capital social entrepreneurial et d'accès aux ressources externes essentielles. La deuxième section sera consacrée à la présentation du modèle de recherche ainsi que des hypothèses de recherche proposées. Dans un troisième temps, nous développerons la méthodologie de recherche adoptée. La présentation et la discussion des résultats feront l'objet de la quatrième section. Finalement, nous mettrons en relief les principales contributions, limites et perspectives de recherches futures au niveau de la conclusion.

\section{FONDEMENTS THÉORIQUES}

Avant de développer les hypothèses de recherche qui traduisent les effets possibles des compétences sociales de l'entrepreneur ainsi que le rôle que pourrait jouer son capital social dans l'obtention des ressources externes clés, nous allons approfondir, dans ce qui suit, notre compréhension des concepts clés de cette recherche, à savoir les compétences sociales, le capital social ainsi que l'accès aux ressources clés requises à la phase de survie-développement d'une entreprise TIC nouvellement créée.

\subsection{L'accès aux ressources externes essentielles : un préalable à la survie- développement d'un projet TIC nouvellement créé}

En réponse aux objectifs de cette étude, une prise en considération de la modélisation de Bruyat (1993), Bruyat et Julien (2001), et Fayolle $(2005,2007)$ a permis d'en appréhender et d'en implémenter le processus de création d'entreprises. Ce processus pourrait être décliné en trois phases successives : le déclenchement, l'engagement et la survie-développement. ${ }^{1}$ Par ailleurs, plusieurs recherches ont souligné que la phase de survie-dévelop-

1 Pour plus de détails sur les phases d'un processus entrepreneurial, voir Omrane, Fayolle et Zeribi (2011). 
pement, caractérisée par l'incertitude, le manque de légitimité, le handicap de la nouveauté et la rareté des ressources, est considérée comme une étape clé et déterminante de la réussite du processus entrepreneurial (Shane et Delmar, 2004; Ravasi et Turati, 2005 ; Tornikoski et Scott, 2007). D'autres travaux ont particulièrement avancé que c'est plutôt le manque de ressources, notamment financières et informationnelles requises au développement des projets entrepreneuriaux, qui menace considérablement leur survie (Ravasi et Turati, 2005 ; Groen, 2005 ; Fayolle, 2007). À cet effet, cette étude se focaliserait sur les ressources préalables au développement d'un nouveau projet de création et qui sont les plus récurrentes au niveau de la littérature. Ces ressources clés concernent les informations technico-commerciales (dénommées aussi professionnelles) ainsi que le financement requis provenant des capitaux-risqueurs. Dans cet ordre d'idées, force est de préciser que ce travail trouve son originalité dans le recours aux travaux de Baron et Tang (2009). Ces auteurs ont mis en relief que, pour assurer la croissance d'un nouveau projet de création, l'entrepreneur aurait besoin d'informations simples, non confidentielles et explicites. Ces informations sont dites professionnelles et se rattachent à des données techniques (rattachées à l'évolution de la technologie) et commerciales (relatives aux besoins et tendances du marché ainsi qu'aux politiques et stratégies des concurrents). Les travaux de Ferrary et Granovetter (2009), Deffains-Crapsky (2011) et Talavera, Kiong et Kiong (2012) ont également servi d'appui mettant en perspective que l'entrepreneur est appelé à diversifier ses sources de financement. De leurs points de vue, si l'attention est plutôt accordée aux actionnaires de proximité (disposant des fonds de proximité) pour assurer le déclenchement, elle serait tournée vers les investisseurs providentiels et les banquiers lors du démarrage. Pendant la $3^{\mathrm{e}}$ étape du développement entrepreneurial, le porteur d'un projet devrait mobiliser davantage des capitaux-risqueurs.

\subsection{Les compétences sociales de l'entrepreneur : définitions et acceptions}

Le concept de compétences sociales a été introduit par Thorndike (1920), puis repris par Marlowe (1986) pour désigner une forme distincte d’intelligence générale.

Un peu plus tard, un courant de recherches en sciences sociales a émergé afin de mieux discerner les compétences sociales et leur impact sur la productivité au travail, la performance organisationnelle et le leadership (Ferris, Witt et Hochwarter, 2001 ; Witt et Ferris 2003).

De nombreux chercheurs, dont Ferris, Davidson et Perrewé (2005), Hochwarter, Treadway, Witt et Ferris (2006), ainsi que Wu et Turban et Cheung (2012), ont avancé que la compétence sociale réfère à la perspicacité interpersonnelle de l'individu et sa capacité d'ajuster son comportement aux différentes demandes situationnelles, ainsi que d'influencer et de contrôler les réponses des autres de façon à satisfaire ses motivations et objectifs prévus.

Dans cette même logique, d'autres auteurs ont mis en perspective qu'un individu qui a su développer des compétences socio-relationnelles et une aptitude à communiquer avec aisance est mieux prédisposé à percevoir et interagir efficacement avec autrui, ce qui lui permet de s'adapter aux différents contextes sociaux pour atteindre ses aspirations (Ferris, Witt et Hochwarter, 2001 ; Ferris, Davidson et Perrewé, 2005 ; Perrewé et al., 2004 ; Perrewé, Ferris, Stoner et Brouer, 2007). 
Dans le champ de l'entrepreneuriat, une perspective de recherche engagée par Markman et Baron (1998), Baron et Brush (1999), Baron et Markman (2000, 2003), Baron (2004b), et Baron et Tang (2009), a consolidé les résultats des travaux antérieurs menés en sciences psychosociales et sociocognitives. De tels chercheurs ont démontré que des compétences sociales spécifiques, comme la perception sociale, l'adaptabilité sociale, l'influence interpersonnelle et l'expressivité, constituent les meilleurs indicateurs de la réussite entrepreneuriale.

(1) La persuasion sociale (ou l'influence interpersonnelle) est définie comme la capacité de l'individu à convaincre et changer les attitudes, comportements et attentes des autres afin de parvenir à un accord préalable à l'atteinte des objectifs visés lors d'une négociation (Cialdini, 2000 ; Dillard et Marshall, 2003 ; Dillard, 2004).

(2) Le management de l'impression renvoie aux travaux abordés respectivement par Bolino et Turnley (1999), Baron et Markman (2003) ainsi que Baron et Tang (2009), et selon lesquels la gestion de l'impression réfère à l'aptitude à induire une impression positive et des réactions favorables chez les autres (Metts et Grohskopf, 2003). De telles études indiquent que le management de l'impression comprend deux composantes : la promotion de soi et les efforts de valorisation d'autrui. La promotion de soi incarne la capacité de l'entrepreneur à mettre l'accent sur ses propres accomplissements, expériences et compétences. Cependant, la valorisation d'autrui représente l'ensemble des efforts et techniques déployés par le porteur d'un projet afin d'exprimer son estime et sa gratitude envers les autres, en mettant en valeur leur retour d'informations et leurs conseils.

(3) L’intelligence émotionnelle consiste à disposer d'habiletés en matière d'automotivation, de contrôle et de gestion de ses propres émotions et de celles des autres (Goleman, 1998 ; Goleman, Boyatzis et Mckee, 2002 ; Bar-On, 2005). Les écrits effectués essentiellement par Goleman $(1998,2001)$ et Goleman, Boyatzis et Mckee (2002) permettent de faire la distinction entre deux composantes de l'intelligence émotionnelle : l'intelligence émotionnelle de soi qui représente la conscience de soi et la gestion de soi, et l'intelligence émotionnelle sociale qui renvoie à la conscience sociale et à la gestion des relations avec les autres.

(4) La perception sociale incarne le processus de compréhension exacte des traits, des motivations et des intentions d'autrui. Elle implique la capacité de « lire » et de comprendre les autres (DePaulo, 1994 ; Baron et Markman, 2003).

(5) L’adaptabilité sociale désigne la capacité de s'adapter à des personnes ayant des backgrounds différents et renvoie à une flexibilité comportementale vis-à-vis de différentes situations sociales (Ferris et al., 2007).

(6) L'expressivité représente l'aptitude d'un individu à exprimer ouvertement ses propres émotions, sentiments et pensées d'une clarté qui génère de l'enthousiasme chez les autres (Cialdini, 2000 ; Baron et Tang, 2009).

\subsection{Le capital social entrepreneurial : vers une approche «bridging»}

Les propos de Baron et Markman $(2000,2003)$ ainsi que ceux de Baron (2004a, b) constituent un apport non négligeable pour la présente étude puisqu'ils permettent d'appréhen- 
der le capital social entrepreneurial, ses différentes mesures et vertus vis-à-vis de la performance de l'organisation. Plus particulièrement, le capital social entrepreneurial est assimilé à l'agrégation des ressources encastrées dans, disponibles par, et dérivées du réseau de relations sociales possédées par un individu ou une organisation (Inpken et Tsang, 2005 ; Kim et Aldrich, 2005 ; Anderson et Jack, 2007).

Par ailleurs, il a été plus judicieux de prendre appui sur l'approche dénommée «bridging social capital perspective » 2 prônée par Burt $(1992,2000)$ et reprise par plusieurs chercheurs à l'instar d'Adler et Kwon (2002). Cette perspective met en valeur l'importance d'un capital social étendu et riche en liens faibles (non affectifs) et diversifiés. Elle soutient que les liens qui sont faibles et structuralement diversifiés sont plus bénéfiques que les liens forts, notamment dans les secteurs turbulents et incertains, tels que le domaine des TIC (Elfring et Hulsink, 2003 ; Bhagavatula, Elfring, Tilburg et Bunt, 2010).

Par réseau étendu, on entend un réseau dont la taille est grande en termes de nombre de contacts (Lin, 2001 ; Greve et Salaff, 2003). Ces contacts peuvent être soit forts, soit faibles.

Opposés aux liens forts caractérisés par un degré de confiance, d'affection et d'attachement interpersonnels élevés (parents et membres de famille, amis intimes, condisciples...), les liens faibles correspondent plutôt aux connaissances vagues, occasionnelles ou professionnelles qui ne découlent pas d'un investissement émotionnel (Dubini et Aldrich, 1991). Dans cette logique, Granovetter (1983) s'emploie minutieusement à démontrer, via sa théorie de " la force des liens faibles », que ces derniers sont plus porteurs de valeur comparativement aux liens forts.

En ce qui a trait à la densité du réseau, elle se rapporte à la proportion de paires de personnes qui sont reliées dans un réseau de relations sociales. Dans cette perspective, Burt (1992, 1997, 2000) a stipulé que : moins le réseau personnel est dense, plus les trous structuraux ${ }^{3}$ qu’il $^{\prime}$ renferme sont nombreux et plus les avantages qui en sont tirés sont considérables. Une autre mesure dérivée de la densité est baptisée « diversité structurale » dénommée désormais DS. Cette mesure constitue un indicateur de la pauvreté du réseau en contacts redondants qui permet d'accéder aux mêmes sources d'information, via le recours aux mêmes personnes (Burt, 1992, 1997).

La qualité des liens sociaux renvoie à l'hétérogénéité des liens sociaux. Dans cette perspective, Lin (2001) a démontré que l'entrepreneur a autant de chances d'accéder aux ressources externes qu'il détient des relations avec des contacts à statut élevé (en termes de catégorie socioprofessionnelle).

2 Burt $(1992,1997)$ ainsi qu'Adler et Kwon (2002) ont proposé deux perspectives du capital social: (1) une perspective «bonding " ou interne (par rapport à l'acteur focal) qui conçoit le capital social en termes de liens forts constituant un réseau dense de relations sociales et (2) une perspective «bridging» ou externe (par rapport à l'acteur focal) qui représente le capital social par les liens faibles, non redondants dont dispose l'individu.

3 Un trou structural est défini en tant qu'un vide entre deux contacts non redondants dans le réseau (Burt, 1992). 


\section{MODĖLE CONCEPTUEL ET HYPOTHĖSES DE RECHERCHE}

Dans la continuité des travaux de Markman et Baron (1998), Baron et Markman (2000, 2003) ainsi que ceux de Baron et Brush (1999), Baron (2004a) et Baron et Tang (2009), le modèle conceptuel proposé pour cette recherche apparaît dans la figure 1 (présentée dans ce qui suit). Il traduit le processus dynamique par lequel les entrepreneurs TIC utilisent et développent leur capital social par le biais des compétences sociales spécifiques dont ils disposent afin d'atteindre leurs objectifs en termes d'accès aux ressources externes requises à la survie-développement de leurs nouvelles entreprises. Afin de contextualiser cette recherche, nous avons préalablement conduit une étude exploratoire auprès de dix entrepreneurs TIC tunisiens et cinq capitaux-risqueurs (via des entretiens semi-directifs, de 30 à 45 minutes chacun). Le choix a été arrêté sur les entrepreneurs qui ont entamé avec succès la phase de survie-développement de leurs nouvelles entreprises créées dans le secteur des TIC. Par ailleurs, tous les acteurs ont été interviewés pendant le dernier trimestre de l'année 2010 et le troisième trimestre de l'année 2011. L'analyse de contenu des données recueillies ${ }^{4}$ auprès des entrepreneurs a bien constitué un appui à la littérature qui a mis en relief que les ressources externes requises pour assurer la survie-développement des projets TIC sont essentiellement les informations technico-commerciales utiles et le financement en provenance des capitaux-risqueurs.

Figure 1. MOdÈLE CONCEPTUEL DES EFFETS DES COMPÉTENCES SOCIALES ET DU CAPITAL SOCIAL DE L'ENTREPRENEUR SUR SON ACCÈS AUX RESSOURCES EXTERNES CLÉS

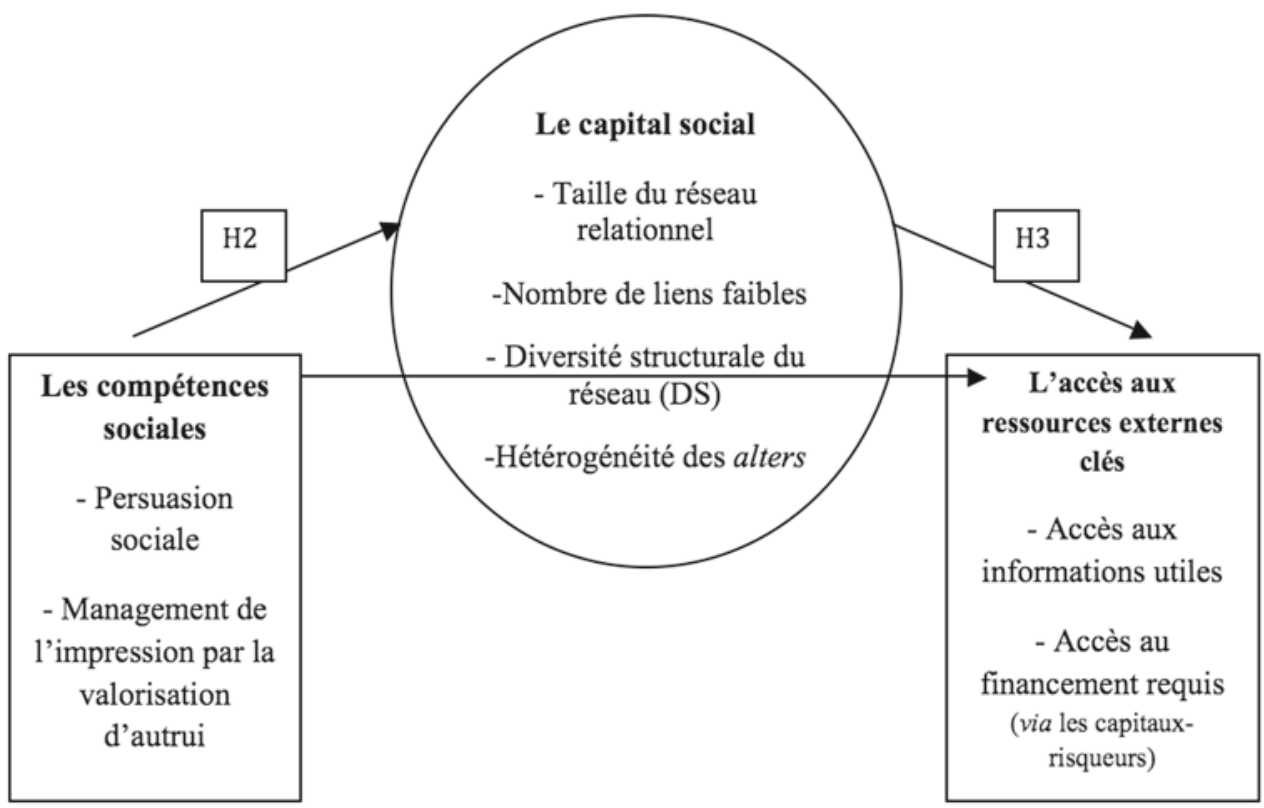

4 Cette analyse de contenu a été faite compte tenu des recommandations proposées par Mukamurera, Lacourse et Couturier (2006), "des avancées en analyse qualitative : pour une transparence et une systématisation des pratiques ». 
Le recoupement des résultats de cette même analyse avec ceux issus des données provenant des capitaux-risqueurs interviewés a également permis de relever trois compétences sociales clés qui sont : la persuasion sociale, l'intelligence émotionnelle de soi et la valorisation d'autrui.

Les hypothèses de recherche qui sont susceptibles de tester les relations entre les compétences sociales de l'entrepreneur, son capital social et son accès aux ressources externes clés, ont été postulées sur la base de deux principaux critères :

- Les résultats de l'étude exploratoire qui a été menée aussi bien auprès des entrepreneurs qu'auprès des capitaux-risqueurs (Omrane, 2013).

- Les principaux travaux empiriques qui traitent, d'une manière ou d'une autre, les relations entre les trois concepts clés de cette étude (pour plus de détails, voir l’annexe 1).

\subsection{Les effets des compétences sociales de l'entrepreneur sur son accès aux ressources externes clés}

En dépit de la rareté des études portant sur l'impact des compétences sociales de l'entrepreneur sur son accès aux ressources externes stratégiques, quelques travaux avancés par Markman et Baron (1998), Baron et Markman (2000, 2003), Baron (2004a) et Baron et Tang (2009) ont été recensés. De tels écrits mettent en relief les retombées positives des habiletés sociales de l'entrepreneur sur son accès aux ressources externes requises au développement de son entreprise nouvelle. L'hypothèse qui suit peut donc être avancée :

Hypothèse 1: les compétences sociales de l'entrepreneur influencent positivement son accès aux ressources externes clés.

Or, comme nous l'avons précédemment mentionné dans la section 1, cette recherche portera sur les compétences sociales qui sont les plus appropriées au contexte TIC tunisien et plus particulièrement à la phase de survie-développement d'une entreprise nouvelle. Ces compétences sont : la persuasion sociale, la gestion de l'impression moyennant les efforts de valorisation des vis-à-vis et l'intelligence émotionnelle de soi.

\subsubsection{L'IMPACT DE L'INFLUENCE SOCIALE DE L'ENTREPRENEUR SUR SON ACCÈS AUX RESSOURCES EXTERNES CLÉS}

Markman et Baron (1998), Baron et Markman (2003), ainsi que Baron et Tang (2009), ont souligné que les entrepreneurs qui ont un style personnel subtil et persuasif sont mieux prédisposés à convaincre les parties prenantes clés de leurs projets, voire de bonifier leurs produits/services. De telles parties prenantes comprennent les investisseurs potentiels, les clients et fournisseurs, avec lesquels le porteur d'un projet devrait aboutir à un consentement préalable afin de développer son entreprise. Ces considérations confortent d'ailleurs les propos de Ferris, Davidson et Perrewé (2005) qui ont mis en valeur la capacité de l'employeur d'influencer ses collaborateurs afin de solliciter leur aide. De ce fait, nous pouvons suggérer les hypothèses suivantes :

Hypothèse 1a: les compétences de l'entrepreneur en matière de persuasion sociale ont un impact positif sur son accès au financement requis de la part des capitauxrisqueurs. 
Hypothèse $1 \mathrm{~b}$ : les compétences de l'entrepreneur en matière de persuasion sociale ont un impact positif sur son accès aux informations utiles au développement de son projet.

\subsubsection{L'IMPACT DES EFFORTS DE VALORISATION D’AUTRUI DÉPLOYÉS PAR L'ENTREPRENEUR SUR SON ACCÈS AUX RESSOURCES EXTERNES CLÉS}

Shepherd et Zacharakis (2001), ainsi que Zacharakis, McMullen et Shepherd (2007), ont démontré que les décisions des capitaux-risqueurs relatives à l'investissement dans les nouveaux projets de création dépendent du profil de l'entrepreneur, de son comportement, de son expérience, ainsi que de sa façon de se vendre et d'induire une réaction positive à leur égard.

Plus spécifiquement, Markman et Baron (1998), Baron et Markman (2003), Metts et Grohskopf (2003), et Baron et Tang (2009) ont mis en relief l'impact positif des efforts de louange d'autrui mobilisés par l'entrepreneur sur son accès aux ressources et informations sollicitées. Nous pouvons donc émettre les deux hypothèses qui suivent :

Hypothèse 1c: l'aptitude de l'entrepreneur à valoriser ses vis-à-vis influence positivement son accès au financement requis.

Hypothèse 1d: l'aptitude de l'entrepreneur à valoriser ses vis-à-vis influence positivement son accès aux informations utiles au développement de son projet.

\subsubsection{L'IMPACT DE L'INTELLIGENCE ÉMOTIONNELLE DE SOI DE L'ENTREPRENEUR SUR SON ACCÈS AUX RESSOURCES EXTERNES CLÉS}

Les travaux de recherche développés par Goleman (2006), Bar-On (2005), Goleman et Boyatzis (2008) et Cavazotte, Moreno et Hickmann (2012) ont largement démontré que l'intelligence émotionnelle de soi d'un leader facilite son adhésion à des individus enclins à lui procurer des ressources externes et renforce en retour sa performance. En nous inscrivant dans ce courant de recherche, les hypothèses suivantes peuvent être émises :

Hypothèse 1e: l'intelligence émotionnelle de soi de l'entrepreneur influence positivement son accès au financement requis auprès des capitaux-risqueurs.

Hypothèse 1f: l'intelligence émotionnelle de soi de l'entrepreneur influence positivement son accès aux informations utiles au développement de son projet.

\subsection{Les effets des compétences sociales de l'entrepreneur sur le développement de son capital social}

En dépit de la croissance du nombre des études qui ont mobilisé la théorie des réseaux sociaux et celle du capital social afin d'appréhender la dynamique de développement des projets entrepreneuriaux (Chabaud et Njigol, 2010), nous avons recensé très peu de travaux empiriques dédiés aux relations susceptibles d'exister entre les compétences sociales de l'entrepreneur et le développement de son capital social. Nous pourrions, tout de même, nous référer à quelques éléments de réflexion qui ont fondé notre propos. En effet, Baron et Markman (2000, p. 1) ont mentionné que «specific social skills, such as the ability to read 
others accurately, make favorable first impressions, adapt to a wide range of social situations, and be persuasive, can influence the quality of these interactions ». Ces mêmes auteurs ont également rajouté que "social capital is often the result of such skills".

Dans cette même ligne de réflexion, Diener et Seligman (2002) ont avancé que les personnes qui ont capitalisé des compétences sociales tendent à avoir des contacts sociaux plus nombreux que les personnes détenant peu d'habiletés relationnelles.

De même, Baron (2004a, p. 222) a révélé que « les compétences sociales peuvent fournir un fondement important pour le développement du capital social».

À partir de ce qui vient d'être évoqué, l'hypothèse suivante peut être postulée :

Hypothèse 2: les compétences sociales de l'entrepreneur influencent positivement le développement de son capital social.

Chi, Fang, Chen et Baron (2008) ont développé un modèle qui a pour objet d'expliquer le processus dynamique par lequel les compétences sociales de l'entrepreneur (à savoir sa sincérité apparente, sa capacité de réseautage, son influence interpersonnelle et son intelligence sociale) lui permettent de mobiliser des réseaux sociaux en vue d'atteindre une meilleure performance entrepreneuriale. Ces propos ont également été confirmés par Zhao, Frese et Giardini (2010).

À cet égard, les hypothèses qui suivent peuvent être suggérées :

Hypothèse 2a: plus l'entrepreneur dispose de compétences en matière de persuasion sociale, plus son capital social est développé.

Hypothèse 2b: plus l'entrepreneur dispose de compétences en matière de valorisation des autres, plus son capital social est développé.

Hypothèse 2c: l'intelligence émotionnelle de soi de l'entrepreneur influence positivement le développement de son capital social.

\subsection{Les effets du capital social de l'entrepreneur sur son accès aux ressources externes clés}

Quelques résultats de recherche ont assimilé le capital social à une ressource productive qui facilite l'action individuelle (Lin, 2001 ; Elfring et Hulsink, 2003), les opérations commerciales (Coleman 1990 ; Burt, 2000) et la création de valeur (Tsai et Ghoshal, 1998 ; Maurer et Ebers, 2006).

Par ailleurs, Van Der Gaag et Snijders (2005), ainsi que Lee, Tüselmann, Jayawarna et Rouse (2011) ont affirmé que les personnes qui ont un capital social développé ont tendance à accumuler plus de ressources. Dans la lignée des travaux de Nahapiet et Ghoshal (1998) et Seibert, Kraimer et Liden (2001), ces mêmes auteurs ont adopté l'approche par les ressources pour expliquer la manière dont le capital social influence le niveau d'éducation, l'accès au financement, et le support social personnel. Dans cette logique, l'hypothèse suivante peut être proposée :

Hypothèse 3: $\quad$ plus le capital social de l'entrepreneur est développé, plus son accès aux ressources externes essentielles est facilité. 
Plus spécifiquement, Shane et Cable (2002), Neergaard et Madsen (2004), Batjargal et Liu (2004), ainsi que Talavera, Xiong et Xiong (2012) ont mis en exergue que les entrepreneurs, qui possèdent un capital social étendu et composé de personnes de références à statuts élevés (dénommés encore des référents), ont plus de chances d'avoir un appui financier provenant des capitaux-risqueurs et des investisseurs potentiels (relativement aux entrepreneurs qui ont un réseau limité de connaissances). L'hypothèse suivante peut être postulée :

Hypothèse 3a: le capital social de l'entrepreneur facilite son accès au financement provenant des capitaux-risqueurs.

De même, Seibert, Kraimer et Liden (2001) ont souligné que l'accès aux informations et aux ressources, ainsi que le parrainage jouent le rôle de médiateur entre le capital social de l'entrepreneur (formé par ses liens faibles et diversifiés) et son succès professionnel. D’autres chercheurs ont mis en exergue que le capital social permet aux entrepreneurs de résoudre les problèmes qu'ils rencontrent par la facilitation du partage de l'information et de la coordination interacteurs (Lazega et Pattison, 2001 ; Yli-Renko, Autio et Sapienza, 2001 ; Maurer et Ebers, 2006 ; Ozgen et Baron, 2007).

Fornoni, Arribas et Vila (2012) ont également mentionné que les relations sociales jouent un rôle important dans le développement des start-ups créées par les entrepreneurs argentins, car elles leur permettent de se procurer des informations de manière efficiente et à des prix économiques. Ainsi, nous pouvons suggérer l'hypothèse testable suivante :

Hypothèse 3b: le capital social de l'entrepreneur facilite son accès aux informations appropriées.

\section{MÉTHODOLOGIE DE RECHERCHE}

L'objectif de cette étude consiste à tester le rôle des compétences sociales de l'entrepreneur dans le développement de son capital social, ainsi que dans son accès aux ressources externes clés. Pour ce faire, nous avons fait une enquête au moyen d'un questionnaire en adoptant la méthodologie présentée dans ce qui suit.

\subsection{Pré-test, échantillon et procédure de collecte des données}

Nous avons combiné les deux approches : qualitative-exploratoire et quantitative-confirmatoire. Comme il a été préalablement indiqué, afin de mieux contextualiser cette étude, nous avons mené une étude qualitative et conduit des entretiens semi-directifs auprès de dix entrepreneurs TIC et cinq capitaux-risqueurs tunisiens.

Compte tenu des résultats obtenus, cette étude s'est limitée aux compétences sociales spécifiques qui ont été les plus prônées par les entrepreneurs TIC interviewés. Ces compétences sont la persuasion sociale, la valorisation d'autrui et l'intelligence émotionnelle de soi. Une première version du questionnaire a donc été développée en langue anglaise.

Pour que chaque échelle de mesure traduise adéquatement le contenu du concept en question, nous nous sommes appuyées sur les travaux de Besson et Haddadj (2003) portant sur 
l'adaptation internationale d'un instrument de mesure (avec l'aide de quatre académiciens spécialisés en management et anglais des affaires). Quelques modifications ont été faites pour éliminer toute contradiction ou changement pouvant affecter le contenu sémantique de certains énoncés. L'enquête finale a été vérifiée suite au pré-test du questionnaire auprès de 6 entrepreneurs. Par la suite, de multiples sources d'information (l'Agence de développement d'industrie et d'innovation, les chambres de commerce et les responsables des communications des parcs technologiques) ont été consultées afin de se constituer un échantillon définitif de 147 entrepreneurs TIC. Cependant, 120 réponses complètes ont été obtenues de la part des porteurs de projets (affichant un taux de réponse de $81 \%$ pendant le $3^{\mathrm{e}}$ trimestre 2011 et le $1^{\text {er }}$ trimestre 2012). Les répondants, basés sur Tunis, Sousse, Sfax et Monastir, sont à $97 \%$ des hommes. Ils ont une moyenne d'âge de 29 ans et détiennent une expérience de deux à trois ans dans le domaine des TIC.

\subsection{Mesures des variables du modèle}

Les mesures des variables ont été adoptées à partir de la littérature. Toutefois, la reformulation de certains items et l'exclusion d'autres étaient nécessaire pour purifier les échelles de mesure et améliorer leur fiabilité, ainsi que la crédibilité des données fournies par les interviewés. L'instrument de mesure finale qui en résulte contient des items clairs et concis (non redondants) qui reflètent le contenu de leurs construits respectifs.

Par ailleurs, les items qui correspondent à chaque construit étaient sujets à l'Analyse en Composantes Principales (ACP) avec la rotation de Varimax 5 . Dans ce cas, la matrice de corrélation inter-items et la qualité de représentation de chaque variable latente ont été examinées afin de purifier les mesures et d'éliminer les indicateurs qui manifestent de faibles contributions factorielles (suite à l'examen des communautés). En outre, les seuls items qui ont été retenus sont ceux qui concourent à une valeur de KMO supérieure à 0,7 et une valeur propre supérieure à 1 pour chaque facteur (relativement aux critères de Kaiser-Mayer-Olkin, Bartlett et Kaiser). Pour quelques facteurs, une valeur de KMO proche de 0,65 a été acceptée.

Un test de la fiabilité, de l'unidimensionnalité de chaque variable latente, ainsi que de la validité convergente et discriminante (via le logiciel SMART PLS) a également été effectué. Pour ce faire, il a été question de vérifier que l'alpha de Cronbach et l'indicateur de fiabilité composite de chaque construit présentent des valeurs supérieures à 0,70 , seuil limite suggéré par Nunnally (1978).

\subsubsection{MESURES DES COMPÉTENCES SOCIALES}

Les échelles de mesure utilisées afin d’opérationnaliser les compétences sociales spécifiques ont été élaborées en référence aux travaux empiriques menés par Markman et Baron (1998), Baron et Markman (2003), Chi et al. (2008), et Baron et Tang (2009). Ces échelles de Likert sont à 5 points (allant de 1 pour « Pas du tout d'accord» à 5 pour « Tout à fait d'accord»). Les scores élevés reflètent des niveaux appréciés de compétences sociales.

5 La rotation Varimax crée des facteurs orthogonaux et non corrélés. 


\section{Opérationnalisation de la persuasion sociale}

Pour mesurer la persuasion sociale, nous nous sommes appuyées sur l'instrument de mesure développé par Markman et Baron (1998). Toutefois, dans le cadre de cette étude, uniquement 3 items convergent vers la persuasion sociale. Ils expliquent $78,667 \%$ de la variance totale $(\mathrm{KMO}=0,734)$. Les deux indicateurs de mesure (4 et 5$)$ ont été faiblement corrélés avec les trois autres items et ont donc été écartés de l'échelle de mesure retenue (voir tableau n ${ }^{\circ} 2$ ).

\section{Opérationnalisation du management de l'impression via la valorisation d'autrui}

L'échelle de mesure (à huit items) proposée par Baron et Tang (2009) a été utilisée pour mesurer le management de l'impression. Dans leur étude, ces chercheurs se sont basés sur l'instrument de mesure auparavant suggéré par Bolino et Turnley (1999), mettant en relief la distinction entre deux construits : la valorisation d'autrui (à quatre items) et la promotion de soi (à quatre items). Suite à l'analyse factorielle que nous avons conduite pour les quatre items correspondants à la valorisation d'autrui, un indicateur de mesure a été faiblement corrélé aux autres items; il a donc été supprimé. Les trois autres items convergent adéquatement vers leur facteur correspondant, enregistrant des valeurs propres supérieures à 1 . Ils expliquent $65,256 \%$ de la variance totale $(\mathrm{KMO}=6,73)$.

\section{Opérationnalisation de l'intelligence émotionnelle de soi}

Plusieurs propositions d'échelles de mesure ont été recommandées par les chercheurs afin d'opérationnaliser l'intelligence émotionnelle. Nous nous sommes rapportées à celles proposées par Goleman (2001), Goleman, Boyatzis et Mckee (2002), Bar-On (2005) pour aboutir à une échelle à quatre items. Un item était peu corrélé aux autres et a été écarté. Les trois items obtenus traduisant un construit unidimensionnel ayant un niveau acceptable de KMO $(\mathrm{KMO}=0,806)$. Ce construit correspond bien à l'intelligence émotionnelle de soi. Il a une valeur propre supérieure à 1 et il explique $87,782 \%$ de la variance totale.

\subsubsection{MESURE DU CAPITAL SOCIAL ENTREPRENEURIAL}

Prenant appui sur les mesures adoptées par Burt $(1992,2000)$ et Seibert, Kraimer et Liden (2001), le capital social a été rapproché à un construit multidimensionnel qui comprend la taille du réseau social de l'entrepreneur, la diversité structurale de ce réseau (DS), le nombre de liens faibles, et l'hétérogénéité des alters qu'il renferme. Nous avons opté pour des mesures métriques relatives aux différentes dimensions du capital social. La taille du réseau de l'entrepreneur correspond au nombre de personnes qui l'ont aidé, d'une manière ou d'une autre, au développement de son projet durant les six derniers mois (une taille du réseau est considérée maximale si elle est égale à dix). À l'encontre des liens forts, les liens faibles représentent des relations purement professionnelles ou occasionnelles et fortuites entre l'entrepreneur et ses connaissances (amis, clients, collègues...). Ces liens faibles sont censés l'aider dans le développement de son entreprise nouvelle. Quant à la diversité des alters, elle est opérationnalisée par un score qui est d'autant plus élevé que l'entrepreneur détient des contacts à statut socioprofessionnel élevé.

La mesure de la DS résulte du logiciel UCINET VI suggéré par Borgatti, Everett et Freeman (2002) et qui calcule la « contrainte structurale» (CS) de chaque réseau entrepreneurial. La DS est dérivée de la CS, selon la formule suivante : DS = 1 CS. 
Une échelle métrique à quatre items a donc été développée afin de mesurer le capital social entrepreneurial.

La taille du réseau, le nombre de liens faibles et la DS forment bien une mesure unidimensionnelle ayant une valeur propre supérieure à 1 et expliquant $77,779 \%$ de la variance totale $(\mathrm{KMO}=0,687)$. L'indicateur de mesure de la qualité (ou hétérogénéité) des liens sociaux a été écarté de l'échelle retenue.

\subsubsection{MESURE DE L'ACCÈS AU FINANCEMENT PROVENANT DES CAPITAUX- RISQUEURS}

En référence à la recherche effectuée par Batjargal et Liu (2004), l'accès au financement via les capitaux-risqueurs a été opérationnalisé par trois items. Tout de même, l'échelle de mesure originale a été légèrement modifiée pour être reportée sur une échelle de Likert à 5 points. Le choix d'homogénéisation entre les différentes échelles de mesure relatives aux différentes variables de cette recherche a été fait par souci de souplesse et de praticité au niveau du traitement statistique des données collectées.

En définitive, les trois items retenus pour mesurer l'accès aux capitaux-risqueurs expliquent $73,830 \%$ de la variance totale $(\mathrm{KMO}=0,705)$.

\subsubsection{MESURE DE L'ACCÈS AUX INFORMATIONS UTILES}

L'accès aux informations utiles a été mesuré sur la base des échelles de mesure, avancées par Lee, Strong, Kahn et Wang (2002), Stvilia, Gasser, Twidale et Smith (2007) et Baron et Tang (2009). Nous sommes parvenues à un outil de mesure à quatre items, compte tenu des retours d'informations enregistrés au niveau de la recherche qualitative auparavant menée.

Ces quatre items retenus permettent d'extraire une seule mesure unidimensionnelle et expliquent $78,294 \%$ de la variance totale $(\mathrm{KMO}=0,835)$.

\section{PRÉSENTATION ET DISCUSSION DES RÉSULTATS}

L'analyse factorielle conduite (par le biais du logiciel SPSS-version 18) afin de raffiner les échelles de mesure a donné lieu à six facteurs interprétables qui représentent les variables choisies pour cette étude. Les résultats de cette analyse mettent en relief que les items représentatifs d'un construit contribuent plus considérablement à ce construit qu'aux autres facteurs. En outre, les variables retenues présentent des valeurs propres supérieures à 1 et expliquent $78,139 \%$ de la variance totale.

\subsection{L'évaluation de la validité et de la fiabilité des mesures (validation du modèle de mesure)}

Pour examiner la fiabilité et la validité convergente et discriminante des différents construits de cette étude, nous avons procédé à une analyse factorielle confirmatoire. Pour ce faire, nous 
avons eu recours à la méthode des équations structurelles (MES), en utilisant la méthode des moindres carrés partiels (PLS), sous le logiciel SMART-PLS (version 2.0). Cette approche, basée sur l'analyse de la variance, est dédiée au test des liens de causalité entre des variables dépendantes et indépendantes d'une manière systématique simple (Urbach et Ahlemann, 2010).

La fiabilité de chaque construit a été vérifiée en calculant l'alpha de Cronbach pour évaluer la consistance interne de l'échelle de mesure aussi bien que la fiabilité composite ${ }^{6}$. Les valeurs d'alpha de Cronbach et celles de la fiabilité composite obtenues pour tous les construits de cette étude sont bien au-dessus de 0,7, la valeur limite recommandée par Chin, Marcolin et Newsted (1996).

TABLEAU 1. VALIDITÉ CONVERgENTE, CONTRIBUTIONS FACTORIELLES ET FIABILITÉ

\begin{tabular}{|c|c|c|c|}
\hline Variables latentes & $\begin{array}{l}\text { Indicateurs } \\
\text { de mesure }\end{array}$ & $\begin{array}{l}\text { Contributions } \\
\text { factorielles }\end{array}$ & T-Student \\
\hline \multirow{3}{*}{$\begin{array}{l}\text { Persuasion sociale }(\alpha=0,864, \\
\text { FC }=0,913, \text { VME }=0,779)\end{array}$} & PS1 & 0,907 & 37,769 \\
\hline & PS2 & 0,923 & 53,424 \\
\hline & PS3 & 0,814 & 16,001 \\
\hline \multirow{3}{*}{$\begin{array}{l}\text { Valorisation d'autrui }(\alpha=0,801 \text {, } \\
\mathrm{FC}=0,881, \mathrm{VME}=\mathbf{0 , 7 1 3})\end{array}$} & VA1 & 0,793 & 8,464 \\
\hline & VA2 & 0,915 & 21,112 \\
\hline & VA3 & 0,821 & 8,659 \\
\hline \multirow{4}{*}{$\begin{array}{l}\text { Intelligence émotionnelle de soi } \\
(\alpha=0,943, \mathrm{FC}=0,960, \mathrm{VME}=0,859)\end{array}$} & IE1 & 0,948 & 89,595 \\
\hline & IE2 & 0,971 & 172,990 \\
\hline & IE3 & 0,958 & 81,595 \\
\hline & IE4 & 0,823 & 31,359 \\
\hline \multirow{3}{*}{$\begin{array}{l}\text { Capital social }(\alpha=0,855, \mathrm{FC}=0,912, \\
\text { VME }=0,777)\end{array}$} & CS1 & 0,926 & 88,653 \\
\hline & $\mathrm{CS} 2$ & 0,911 & 58,918 \\
\hline & CS3 & 0,802 & 23,320 \\
\hline \multirow{3}{*}{$\begin{array}{l}\text { Accès aux ressources financières } \\
(\alpha=0,822, \mathrm{FC}=0,893, \mathrm{VME}=0,737)\end{array}$} & AF1 & 0,870 & 23,835 \\
\hline & AF2 & 0,851 & 19,010 \\
\hline & AF3 & 0,854 & 16,888 \\
\hline \multirow{4}{*}{$\begin{array}{l}\text { Accès aux informations utiles } \\
(\alpha=0,906, \mathrm{FC}=0,934, \mathrm{VME}=\mathbf{0 , 7 8 0})\end{array}$} & AI1 & 0,895 & 50,324 \\
\hline & $\mathrm{AI} 2$ & 0,921 & 49,564 \\
\hline & $\mathrm{AI} 3$ & 0,905 & 56,215 \\
\hline & AI4 & 0,809 & 21,973 \\
\hline
\end{tabular}

La validité convergente désigne le degré auquel les différents items convergent uniquement vers leurs construits respectifs, relativement aux items mesurant des construits différents (Henseler, Ringle et Sinkovics, 2009 ; Urbach et Ahlemann, 2010). Un critère communément appliqué est celui de la variance moyenne extraite (VME) proposée par Fornell et Larcker (1981). La valeur-

6 La consistance interne d'une échelle de mesure suppose que tous les indicateurs soient équiprobablement fiables (Cronbach, 1951). Par contre, la fiabilité composite prend en considération le fait que les indicateurs ont des contributions factorielles différentes (Henseler, Ringle et Sinkovics, 2009). 
seuil proposée par ces chercheurs est de 0,5 ; ainsi, la VME devrait être supérieure à 0,5 . En outre, Chin (1998) et Chin, Marcolin et Newsted (2003) indiquent qu'il faudrait vérifier à quel degré la variance des indicateurs est-elle expliquée par la variable latente correspondante. Ceci implique que les valeurs des contributions factorielles d'un construit doivent être supérieures à 0,7 , avec un niveau significatif de T-Student (supérieur à 1,96) (Gefen et Straub, 2005). Dans le cadre de cette étude, la VME de chaque variable latente est bien supérieure à 0,5 et ses contributions factorielles dépassent bien 0,7 , avec une valeur de T-Student significative.

Le tableau 1 expose les contributions factorielles des indicateurs de mesure, la fiabilité, ainsi que la variance moyenne extraite des différentes variables latentes (voir également annexe $\left.\mathrm{n}^{\circ} 2\right)$.

Quant à la validité discriminante, elle traduit le degré auquel les construits diffèrent les uns des autres (Zhu, Kraemer, Gurbaxani et Xu, 2006). Dans l'approche PLS, deux critères sont généralement employés : le critère de Fornell et Larcker (1981) et celui des contributions factorielles croisées dénommées « cross-loadings » proposé par Chin (1998b).

Le premier critère exige d'un construit de partager plus de variances avec ses indicateurs assignés qu'avec n>importe quel autre construit. Ainsi, la racine carrée de la VME de chaque construit devrait être supérieure à la corrélation entre n'importe quelle paire d'autres construits (Gefen et Straub, 2005). Dans la présente recherche, nous avons abouti à une racine carrée de la VME (éléments situés sur la diagonale de la matrice de corrélation) plus grande que la valeur absolue des corrélations inter-construits (éléments situés en dessous de la diagonale).

Selon le deuxième critère, les contributions des items de mesure de chaque construit devraient être supérieures à n'importe quelle contribution d'un autre construit. En d'autres termes, chaque construit converge plus considérablement avec ses propres indicateurs de mesure (Chin, 1998b ; Gefen et Straub, 2005).

Le tableau 2 présente la matrice de corrélation des différentes variables latentes.

Tableau 2. Validité discriminante (1) : Matrice DE Corrélation Des Variables LATENTES

\begin{tabular}{lllllll}
\hline Variables latentes & 1 & 2 & 3 & 4 & 5 & 6 \\
\hline 1-Persuasion sociale & $\mathbf{0 , 8 8 2}$ & & & & & \\
\hline 2-Valorisation d'autrui & 0,371 & $\mathbf{0 , 8 4 4}$ & & & & \\
\hline 3-Intelligence émotionnelle de soi & 0,166 & $-0,027$ & $\mathbf{0 , 9 2 6}$ & & & \\
\hline 4-Capital social entrepreneurial & 0,253 & 0,026 & 0,671 & $\mathbf{0 , 8 8 1}$ & & \\
\hline 5-Accès aux ressources financières & 0,256 & 0,155 & 0,102 & 0,295 & $\mathbf{0 , 8 5 8}$ & \\
\hline 6-Accès aux informations utiles & 0,335 & 0,269 & 0,384 & 0,428 & 0,145 & $\mathbf{0 , 8 8 3}$ \\
\hline Moyenne & $\mathbf{7 , 6 3}$ & $\mathbf{7 , 4 3}$ & $\mathbf{1 0 , 6 5}$ & $\mathbf{6 , 4 5 3}$ & $\mathbf{8 , 2 8}$ & $\mathbf{1 2 , 4 8}$ \\
\hline Écart-type & $\mathbf{2 , 5 8}$ & $\mathbf{2 , 0 2}$ & $\mathbf{3 , 4 2 7}$ & $\mathbf{2 , 9 4 2}$ & $\mathbf{2 , 3 5 9}$ & $\mathbf{3 , 7 5 5}$ \\
\hline
\end{tabular}

Notes : Éléments de la diagonale $=$ la racine carrée de la VME;

Éléments placés en dessous des éléments de la diagonale = corrélations inter-construits. 
Dans le cadre de cette étude, les valeurs des contributions factorielles de chaque variable latente sont supérieures aux valeurs des contributions factorielles croisées avec n'importe quelle autre variable. La validité discriminante est donc vérifiée telle que le démontre le tableau 3.

Tableau 3. Validité discriminante (2) : Matrice de structure des contributions FACTORIELLES ET DES CONTRIBUTIONS FACTORIELLES CROISÉES

\begin{tabular}{|c|c|c|c|c|c|c|}
\hline \multirow{2}{*}{ Mesures } & \multicolumn{6}{|c|}{ Contributions factorielles } \\
\hline & 1 & 2 & 3 & 4 & 5 & 6 \\
\hline \multicolumn{7}{|c|}{ 1-Persuasion sociale (PS) } \\
\hline PS1 & 0,907 & 0,321 & 0,186 & 0,247 & 0,187 & 0,341 \\
\hline PS2 & $\mathbf{0 , 9 2 2}$ & 0,340 & 0,148 & 0,229 & 0,288 & 0,367 \\
\hline $\mathrm{PS} 3$ & 0,814 & 0,336 & 0,081 & 0,182 & 0,184 & 0,084 \\
\hline \multicolumn{7}{|c|}{ 2-Valorisation d'autrui (VA) } \\
\hline VA1 & 0,299 & 0,792 & $-0,003$ & 0,031 & 0,182 & 0,176 \\
\hline VA2 & 0,354 & 0,915 & $-0,001$ & 0,055 & 0,147 & 0,288 \\
\hline VA3 & 0,273 & $\mathbf{0 , 8 2 0}$ & $-0,087$ & $-0,047$ & 0,040 & 0,195 \\
\hline \multicolumn{7}{|c|}{ 3-Intelligence émotionnelle de soi (IE) } \\
\hline IE1 & 0,127 & $-0,034$ & 0,948 & 0,621 & 0,070 & 0,380 \\
\hline IE2 & 0,132 & $-0,040$ & 0,971 & 0,646 & 0,082 & 0,360 \\
\hline IE3 & 0,151 & $-0,055$ & 0,957 & 0,630 & 0,096 & 0,367 \\
\hline IE4 & 0,210 & 0,034 & $\mathbf{0 , 8 2 3}$ & 0,589 & 0,133 & 0,313 \\
\hline \multicolumn{7}{|c|}{ 4- Capital social entrepreneurial (CS) } \\
\hline $\mathrm{CS} 1$ & 0,259 & 0,074 & 0,566 & 0,926 & 0,373 & 0,417 \\
\hline $\mathrm{CS} 2$ & 0,152 & $-0,041$ & 0,767 & 0,911 & 0,085 & 0,374 \\
\hline CS3 & 0,272 & 0,041 & 0,409 & $\mathbf{0 , 8 0 2}$ & 0,345 & 0,336 \\
\hline \multicolumn{7}{|c|}{ 5-Accès aux ressources financières $(\mathrm{AF})$} \\
\hline AF1 & 0,197 & 0,186 & 0,070 & 0,207 & $\mathbf{0 , 8 7 0}$ & 0,112 \\
\hline AF2 & 0,195 & 0,090 & 0,109 & 0,314 & 0,851 & 0,096 \\
\hline AF3 & 0,266 & 0,132 & 0,079 & 0,227 & $\mathbf{0 , 8 5 3}$ & 0,166 \\
\hline \multicolumn{7}{|c|}{ 6-Accès aux informations utiles (AI) } \\
\hline AI1 & 0,390 & 0,260 & 0,430 & 0,455 & 0,130 & 0,894 \\
\hline AI2 & 0,321 & 0,317 & 0,283 & 0,361 & 0,181 & 0,921 \\
\hline AI3 & 0,350 & 0,190 & 0,307 & 0,332 & 0,141 & 0,904 \\
\hline $\mathrm{AI} 4$ & 0,041 & 0,153 & 0,314 & 0,341 & 0,037 & $\mathbf{0 , 8 0 8}$ \\
\hline
\end{tabular}

\subsection{Le test des hypothèses de recherche (estimation du modèle de structure)}

L'estimation du modèle de structure passe par l'analyse des relations se manifestant entre les variables explicatives (les compétences sociales) et les variables à expliquer (le capital social de l'entrepreneur et son accès aux ressources clés) comme le démontre le tableau 4. 
TABleAu 4. SynthèSE DES COEFFICIENTS DE RÉGRESSION DU MODÈLE DE STRUCTURE ET DES STATISTIQUeS PLS

\begin{tabular}{lllll}
\hline Variables latentes & $\begin{array}{l}\text { 4-Capital } \\
\text { social }\end{array}$ & $\begin{array}{l}\text { 5-Accès au } \\
\text { financement }\end{array}$ & $\begin{array}{l}\text { 6-Accès aux } \\
\text { informations } \\
\text { utiles }\end{array}$ & $\begin{array}{l}\text { Hypothèses } \\
\text { de recherche }\end{array}$ \\
\hline $\begin{array}{l}\text { 1-Persuasion } \\
\text { sociale }\end{array}$ & $0,150^{* *}$ & $0,162^{* *}$ & $0,163^{* * *}$ & $\begin{array}{l}\text { Hypothèses } 2 \mathrm{a}, 1 \mathrm{a} \text { et } \\
\text { b confirmées }\end{array}$ \\
\hline $\begin{array}{l}\text { 2-Valorisation } \\
\text { d'autrui }\end{array}$ & $-0,012^{\mathrm{ns}}$ & $0,081^{\mathrm{ns}}$ & $0,207^{* *}$ & $\begin{array}{l}\text { *Hypothèse } 1 \mathrm{~d} \\
\text { confirmée } \\
* \text { Hypothèses 1c } \\
\text { et 2b non vérifiées }\end{array}$ \\
\hline $\begin{array}{l}\text { 3-Intelligence } \\
\text { émotionnelle de } \\
\text { soi }\end{array}$ & $0,646^{*}$ & $-0,167^{\mathrm{ns}}$ & $0,194^{* * *}$ & $\begin{array}{l}\text { *Hypothèses 1f } \\
\text { et 2c confirmées } \\
* \text { Hypothèse 1e } \\
\text { rejetée }\end{array}$ \\
\hline 4- Capital social & & & $\begin{array}{l}\text { Hypothèses 3a } \\
\text { et 3b confirmées }\end{array}$ \\
\hline R & & $0,364^{*}$ & $0,251^{* * *}$ & \\
\hline GOF & 0,472 & 0,144 & 0,2925 & \\
\hline
\end{tabular}

Notes : ns : paramètre non significatif ; ${ }^{*}$ : paramètre significatif à $\mathrm{p}<1 \%$;

${ }^{* *}$ : paramètre significatif à $\mathrm{p}<5 \% ;{ }^{* * *}$ : paramètre significatif à $\mathrm{p}<10 \%$.

Les résultats obtenus confortent nos prévisions relatives aux relations positives existant entre les compétences sociales de l'entrepreneur (sa persuasion sociale, ses efforts de valorisation d'autrui et son intelligence émotionnelle de soi) et son accès aux informations utiles (les valeurs de $\beta$ sont respectivement de 36,4 \%, 20,7 \% et 19,4\%). Les hypothèses $1 \mathrm{~b}$, $1 \mathrm{~d}$ et $1 \mathrm{f}$ sont alors validées. Ce résultat conforte, en partie, les propos avancés par Baron et Tang (2009).

Or, sur les trois compétences sociales retenues dans notre recherche, seulement la persuasion sociale semble exercer une influence positive sur l'accès au financement ( $\beta=16,2 \%)$. L'hypothèse la est alors confirmée. Ce résultat concorde avec ceux obtenus dans les travaux de Baron et Brush (1999) et Baron et Markman (1998, 2003).

Néanmoins, par contre, les hypothèses $1 c$ et le ne sont pas vérifiées. En effet, l'impact de l'intelligence émotionnelle de soi de l'entrepreneur sur son accès au financement est non significatif, alors que l'habileté de l'entrepreneur en valorisation des vis-à-vis est faiblement corrélée à son accès au financement et que les deux valeurs liées à ces compétences sociales n'ont pas atteint la signification statistique. L'accès de l'entrepreneur aux ressources financières est donc facilité essentiellement par la persuasion sociale.

Ces résultats ne sont pas surprenants, car l'accès au financement dépend définitivement des décisions des capitaux-risqueurs et de leur processus d'analyse mental. Ces investisseurs financiers prennent le risque et acceptent d'investir dans un nouveau projet si et seu- 
lement si l'affaire s'avère être du "gagnant gagnant ». À cet effet, les entrepreneurs doués qui font de leur mieux pour fournir des arguments logiques, par leur capacité à attirer des personnes clés comme les capitaux-risqueurs, sont plus enclins à convaincre aisément les investisseurs et induire des réactions positives chez eux (Shane et Cable, 2002 ; Batjargal et Liu, 2004 ; Zacharakis, McMullen et Shepherd, 2007).

Par ailleurs, les résultats atteints mettent en évidence que la persuasion sociale et l'intelligence émotionnelle de soi de l'entrepreneur sont positivement et significativement liées au développement de son capital social, soulignant un effet considérable de l'intelligence émotionnelle de soi $(\beta=64,6 \%$, comparativement à celui de la persuasion sociale $(\beta=15 \%)$. Les hypothèses $2 \mathrm{a}$ et $2 \mathrm{c}$ sont alors corroborées, alors que l'hypothèse $2 \mathrm{~b}$ relative à l'impact des efforts de valorisation d'autrui sur le développement du capital social est rejetée. Subséquemment, les efforts de valorisation d'autrui déployés par l'entrepreneur n'exercent aucun effet sur le développement de son capital social. Dans le contexte tunisien des TIC, il semble que des parties prenantes clés, telles que les investisseurs, clients, fournisseurs ou autres professionnels, ne sont pas assez tentées par la flatterie et les tactiques de louange engagées par l'entrepreneur. Ces personnes sont emportées par les compétences entrepreneuriales en matière d'influence interpersonnelle et d'intelligence émotionnelle de soi. À cet égard, il serait pertinent de souligner que l'intelligence émotionnelle de soi de l'entrepreneur, liée à sa confiance en soi, son auto-efficacité, son contrôle émotionnel de soi et son auto-détermination, contribue à l'extension de son capital social ( $\beta=64,6 \%)$. Ceci signifie que plus l'entrepreneur est enthousiaste et plus il est émotionnellement intelligent, plus le nombre de contacts (essentiellement faibles et diversifiés) faisant partie de son réseau relationnel est élevé.

En outre, les résultats avancés mettent en exergue que le capital social de l'entrepreneur influence positivement son accès aussi bien au financement $(\beta=36,4 \%$ ), qu'aux informations utiles $(\beta=25,1 \%)$. Par conséquent, les hypothèses $3 \mathrm{a}$ et $3 \mathrm{~b}$ sont vérifiées ; ce qui permet de confirmer les propos précédents selon lesquels les liens faibles et diversifiés détenus par un entrepreneur impactent positivement son accès aux informations utiles et riches, voire à des connaissances partagées (Lin, 2001 ; Seibert, Kraimer et Liden, 2001 ; Yli-Renko, Autio et Sapienza, 2001 ; Cantner et Stuetzer, 2010). De plus, à la différence des études antérieures qui ont mis en valeur le rôle des liens forts de l'entrepreneur dans son obtention du financement requis (Shane et Cable, 2002 ; Batjargal et Liu, 2004), cette recherche a mis en évidence que les liens faibles et non redondants du réseau de l'entrepreneur concourent également à son accès aux ressources financières requises pour le développement de sa nouvelle entreprise, de la part des capitaux-risqueurs.

Ainsi, à l'issue des résultats obtenus au niveau de cette étude, nous pourrions en déduire que le capital social de l'entrepreneur joue un rôle médiateur partiel ${ }^{7}$ entre : (a) sa persuasion sociale et son accès aux ressources externes clés (à savoir l'acquisition d'un financement émanant des capitaux-risqueurs et l'obtention des informations requises) et (b) son intelligence émotionnelle de soi et son accès aux informations requises.

7 Pour plus de détails sur la médiatisation partielle, voir Baron et Kenny (1986) et Caceres et Vanhamme (2003). 
Afin d'expliquer la variance des variables latentes endogènes, nous avons procédé au calcul du coefficient de détermination $\left(\mathrm{R}^{2}\right)$ relativement au capital social, à l'accès aux informations utiles et à l'accès au financement.

$\mathrm{R}^{2}$ du capital social entrepreneurial est de 0,472 , ce qui signifie que les compétences sociales expliquent $47,2 \%$ de la variance du capital social. $\mathrm{R}^{2}$ pour l'accès au financement a une valeur de 0,144, tandis que $\mathrm{R}^{2}$ associé à l'accès aux informations utiles est de 0,292. Selon Chin (1998b) et Ringle (2004), les valeurs autour de 0,19, 0,333 et 0,67 sont considérées respectivement comme faibles, modérées et élevées. Ainsi, le coefficient de détermination associé au capital social est élevé, celui de l'accès aux informations utiles est modéré, tandis que celui relatif au financement est faible. La faible valeur de $\mathrm{R}^{2}$ associé à l'accès au financement pourrait incomber au fait que ce sont les liens forts qui influencent, plus que les liens faibles, l'obtention de l'entrepreneur du financement requis. Ceci pourrait expliquer davantage en quoi la majorité des recherches consacrées à l'étude du capital social entrepreneurial dans sa relation avec l'accès aux ressources financières ont opérationnalisé le capital social par le nombre de liens forts et affectifs qu'il renferme (Shane et Shane, 2002 ; Batjargal et Liu, 2004).

Nous trouvons également un indicateur d'ajustement global (ou goodness of fit - abrégé GOF) de 0,483 , ce qui signifie que le pouvoir d'explication global du modèle est élevé. Dans ce sens, Henseler, Ringle et Sinkovics (2009) avancent que des valeurs autour de 0,1, 0,25 et 0,36 sont respectivement considérées comme faibles, modérées et élevées.

Dans l'ensemble, les résultats trouvés mettent en évidence que les entrepreneurs socialement compétents sont plus prédisposés à développer aisément leur capital social. Ce capital-connaissances est largement mobilisé par les entrepreneurs afin d'accéder aux ressources essentielles externes requises à la survie/développement des entreprises nouvellement créées.

\section{CONCLUSION, CONTRIBUTIONS, LIMITES ET VOIES DE RECHERCHE}

Les résultats de cette recherche présentent plusieurs apports à deux niveaux d'analyse :

À un macro-niveau d'analyse, cette étude permet d'appréhender le processus par lequel les entrepreneurs mobilisent leurs compétences sociales et leurs réseaux relationnels afin d'accéder aux ressources requises à une étape problématique du processus entrepreneurial : la survie-développement. À ce stade, les entrepreneurs rencontrent plusieurs problèmes liés à l'asymétrie de l'information, l'incertitude, la rareté de ressources et le manque de légitimité. Ils ont besoin de faciliter leur accès aux personnes clés, voire aux ressources qui sont rares, mais essentielles pour l'acquisition d'une certaine légitimité et crédibilité autour de la viabilité de leurs projets.

À un micro-niveau d'analyse, alors que les recherches antérieures menées essentiellement par Markman et Baron (1998); Baron et Brush (1999); Baron et Markman (2000, 2003); Baron (2004a, b) et Baron et Tang (2009) (dans des contextes nord-américains, anglais et chinois) ont fait l'éloge des effets positifs des compétences sociales sur le succès finan- 
cier des entrepreneurs, cette étude recentre l'attention sur la problématique d'accès aux ressources clés sollicitées durant la survie-développement des nouvelles entreprises. En d'autres termes, cette recherche met en lumière le rôle que jouent les compétences sociales dans l'accès aux ressources externes essentielles. Ces ressources renferment aussi bien les informations utiles que le financement requis dans le contexte - TIC - tunisien.

Par ailleurs, cette présente étude rajoute de nouveaux éléments aux travaux précédemment menés. Elle explore le mécanisme par lequel des compétences sociales spécifiques à savoir la persuasion sociale, l'intelligence émotionnelle de soi et la valorisation d'autrui aident les entrepreneurs à développer leur capital social. En effet, les résultats empiriques obtenus consolident les propos théoriques avancés par Baron et Brush (1999) et Markman et Baron (1998), et Baron et Markman (2000, 2003) selon lesquels les entrepreneurs qui sont socialement compétents sont plus enclins à détenir des réseaux relationnels développés (axés sur un nombre élevé de liens faibles et diversifiés). Dans cette même ligne de réflexion, nous avons tenté de démontrer que les retombées positives générées par le capital social entrepreneurial en termes d'accès aux ressources clés sont considérables pendant la phase de survie-développement d'une entreprise nouvelle (aussi bien que durant les phases antérieures du processus entrepreneurial). Plus particulièrement, la persuasion sociale et le développement d'un répertoire téléphonique bien garni de connaissances constituent des variables clés à l'obtention du financement. La valorisation d'autrui et l'intelligence émotionnelle semblent avoir une portée moins considérable.

Ainsi, la révélation suggérée par Baron et Markman (2003), en ce que « social capital may well exert its primary impact early in the process [...] whereas the effects of social skills in interacting may persist and continue to shape the nature of entrepreneurs' relations with such persons on a long-term basis", pourrait être enrichie.

Nous pensons que cette étude présente, tout de même, quelques limites :

En premier lieu, il existe un biais inhérent à l'adaptation de quelques mesures relatives aux compétences sociales et au capital social dans le sens où les compétences sociales ont été opérationnalisées via une échelle de Likert reportant les réponses accordées par les experts au niveau de la recherche qualitative. En outre, alors que le capital social a été mesuré en référence aux liens faibles, professionnels et diversifiés développés tout au long du processus entrepreneurial, il a été représenté, dans d'autres travaux, par le nombre de liens forts et denses, mettant au point leurs effets sur le succès financier entrepreneurial (Hansen, 1999; Jensen et Greve, 2002 ; Inpken et Tsang, 2005). D’autres chercheurs comme Anderson, Park et Jack (2007) ont assimilé le capital social à un artéfact résultant des interactions sociales représenté par une mise en commun de bienveillance, d'interdépendance et d'échange. Lochner, Kawachi et Kennedy (1999) ont plutôt opérationnalisé le capital social par quatre concepts clés : l'efficacité collective, la cohésion de voisinage, la compétence communautaire et le sens psychologique de la communauté. Notre choix des mesures du capital social se justifie par le fait que, dans le cadre de cette étude, l'attention est focalisée sur la phase de survie-développement des nouveaux projets TIC. En cela, il a été question de prendre en considération trois critères clés : structure (taille du capital social et diversité structurale), nature (nombre de liens faibles) et qualité des liens (leur hétérogénéité).

Il serait donc intéressant de clarifier en quoi nos mesures du capital social et celles de Batjargal et Liu (2004) diffèrent, mais se complètent relativement à leurs effets sur l'accès 
de l'entrepreneur aux capitaux requis. De façon similaire, d'autres chercheurs ont souligné que le capital social devrait être estimé par le mix des liens forts et faibles qu'il comprend (Hite et Hesterly, 2001 ; Elfring et Hulsink, 2003).

Une première perspective de recherche pourrait, par conséquent, être adressée à une jonction entre les liens forts et faibles afin d'évaluer leur influence sur le succès entrepreneurial.

Dans la même perspective, les ressources ont été mesurées par les informations technicocommerciales et le financement provenant des capitaux-risqueurs. La délimitation de ces ressources a été faite compte tenu des résultats de l'étude exploratoire qui convergent, d'ailleurs, avec les présomptions de la littérature.

En deuxième lieu, une autre limite empirique pourrait concerner la période pendant laquelle les données ont été rassemblées, puis traitées. Cette recherche a été conduite dans un laps de temps bien défini, ce qui ne nous a pas permis d'évaluer le niveau des compétences sociales et du capital social entrepreneurial atteints à des périodes distinctes et successives dans le temps.

Il serait donc judicieux d'effectuer une analyse longitudinale pour faire le suivi de l'évolution concomitante du niveau du capital social et de celui des ressources acquises, adéquatement au développement des compétences sociales de l'entrepreneur tout au long du processus entrepreneurial.

En troisième lieu, les résultats atteints se rapportent uniquement au secteur des TIC tunisien (représenté majoritairement par des entrepreneurs de sexe masculin). Nous ne pouvons, toutefois, nous prononcer sur l'adaptation des compétences sociales spécifiques déployées dans le cadre de cette étude (notamment la persuasion sociale, l'intelligence émotionnelle de soi et la valorisation d'autrui) à d'autres contextes culturellement différents. Ainsi, une voie de recherche future pourrait être dédiée à la réplication du test d'un modèle de recherche intégrateur ${ }^{8}$ à d'autres variables, à d'autres phases du processus entrepreneurial (déclenchement, engagement), et/ou à d'autres secteurs (tels que l'agriculture, l'industrie et/ou la biotechnologie), voire à d'autres pays.

Aussi, serait-il intéressant d'offrir des programmes de conseil et de formation aux entrepreneurs non seulement pour les prévenir des erreurs sociales susceptibles de menacer l'atteinte de leurs objectifs visés, mais aussi afin d'augmenter leurs aptitudes d'interaction sociale et de communication avec des personnes à cultures différentes. Ces programmes d'enseignement de l'entrepreneuriat devraient miser davantage sur les techniques déployées pour développer la persuasion sociale, l'intelligence émotionnelle et le management de l'impression, quitte à promouvoir le succès professionnel.

8 Par modèle de recherche intégrateur, nous visons un modèle qui intègre les six domaines de compétences sociales : la persuasion sociale, le management de l'impression, l'intelligence émotionnelle, la perception sociale, l'adaptabilité sociale et l'expressivité. Une autre voie de recherche susceptible d'être étudiée concernerait l'étude de l'impact que pourrait avoir également le CSE sur le développement des compétences sociales (voir Burt, 2010). 


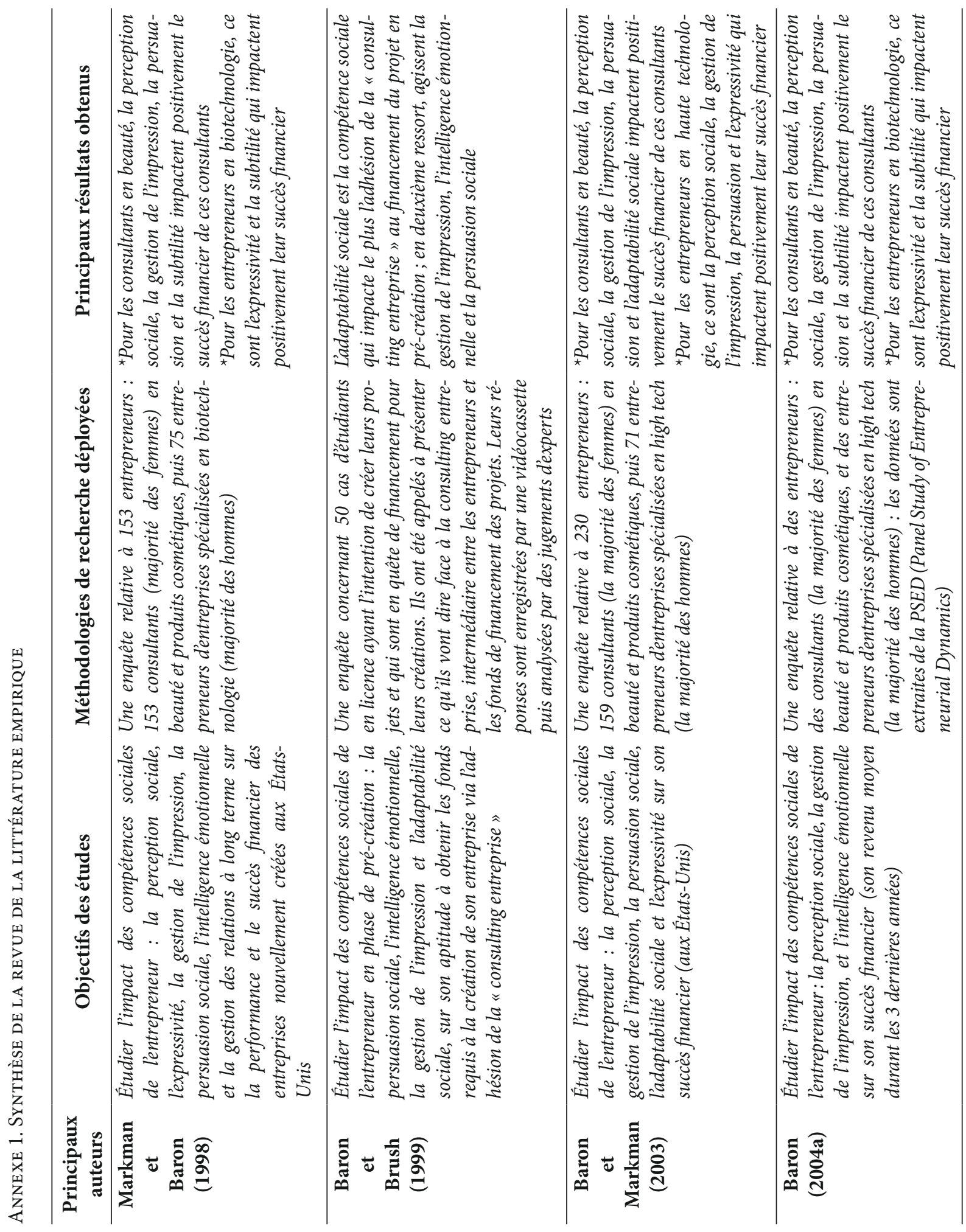




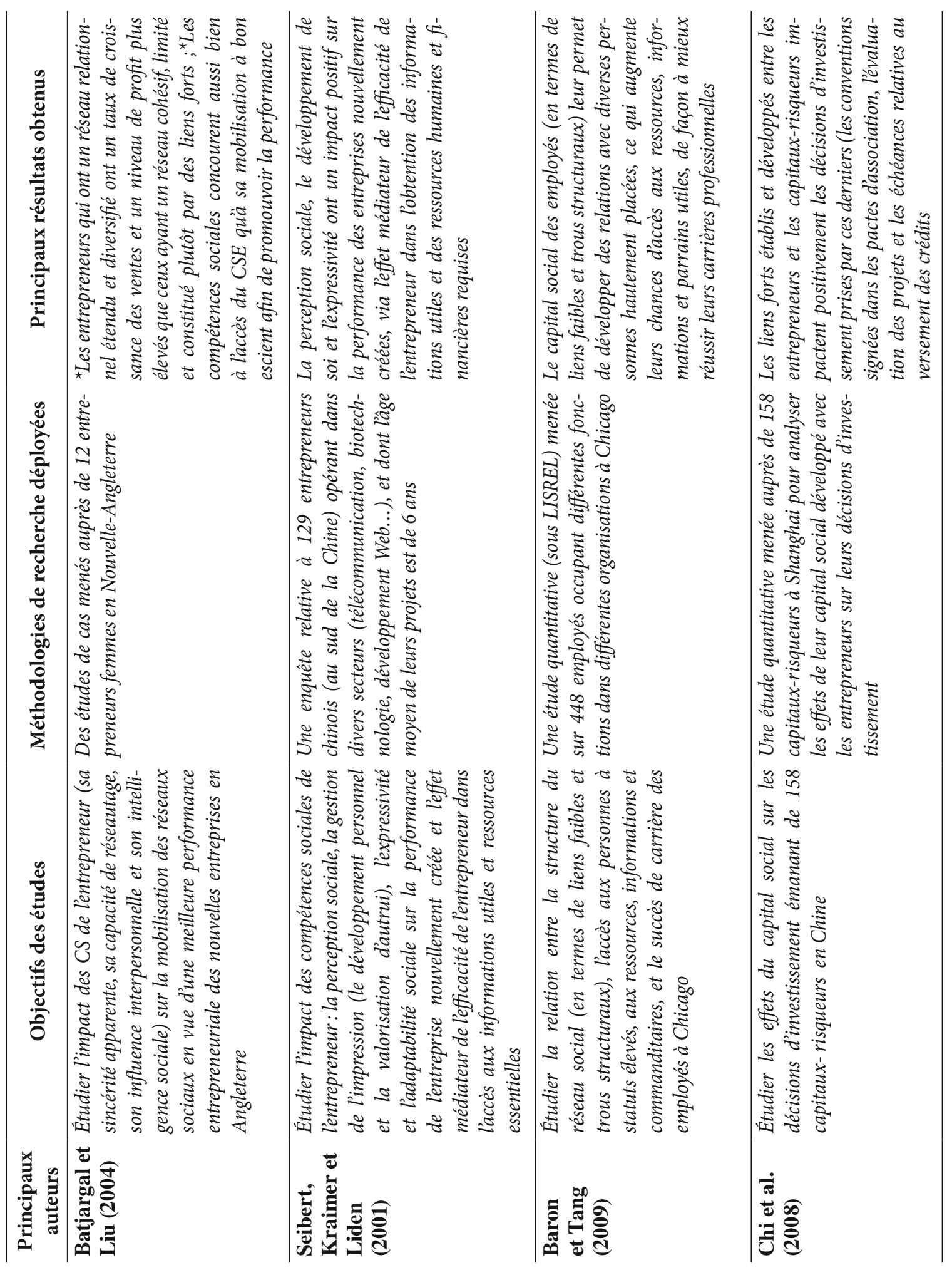




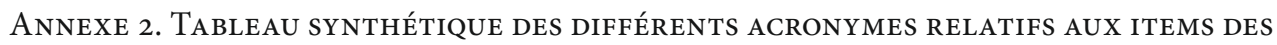
VARIABLES DE L'ÉTUDE

\begin{tabular}{|c|c|c|}
\hline Variables & Items & Acronymes appropriés \\
\hline \multirow[t]{3}{*}{$\begin{array}{l}\text { Persuasion } \\
\text { sociale }\end{array}$} & PS1 & $\begin{array}{l}\text { Je ne trouve aucune difficulté dans la prise de parole devant un } \\
\text { grand public }\end{array}$ \\
\hline & PS2 & $\begin{array}{l}\text { Si j'envisage de persuader quelqu'un de changer ses points de } \\
\text { vue, je réussis le plus souvent à le faire }\end{array}$ \\
\hline & PS3 & $\begin{array}{l}\text { Je peux facilement obtenir l'adhésion des autres concernant ce } \\
\text { que je fais dans la plupart des situations }\end{array}$ \\
\hline \multirow[t]{3}{*}{$\begin{array}{l}\text { Valorisation } \\
\text { d'autrui }\end{array}$} & VA1 & $\begin{array}{l}\text { Je complimente les autres pour qu'ils m'apprécient et me voient } \\
\text { agréable }\end{array}$ \\
\hline & VA2 & $\begin{array}{l}\text { J'accorde des faveurs personnelles aux autres afin de leur } \\
\text { prouver que je suis sympathique }\end{array}$ \\
\hline & VA3 & $\begin{array}{l}\text { Je fais l'éloge des autres à ce qu'ils me considèrent comme une } \\
\text { personne agréable }\end{array}$ \\
\hline \multirow{4}{*}{$\begin{array}{l}\text { Intelligence } \\
\text { émotionnelle } \\
\text { de soi }\end{array}$} & IE1 & $\begin{array}{l}\text { Je comprends et reconnais mes propres émotions et leurs effets, } \\
\text { mes forces ainsi que mes capacités }\end{array}$ \\
\hline & IE2 & $\begin{array}{l}\text { Je contrôle souvent mes émotions les plus perturbatrices, ainsi } \\
\text { que mes impulsions }\end{array}$ \\
\hline & IE3 & $\begin{array}{l}\text { J'actualise souvent mes potentialités et je fais de mon mieux } \\
\text { pour atteindre mes objectifs visant un standard d'excellence }\end{array}$ \\
\hline & IE4 & $\begin{array}{l}\text { Je suis apte à adapter et ajuster mes sentiments conformément } \\
\text { aux différentes situations }\end{array}$ \\
\hline \multirow{3}{*}{$\begin{array}{l}\text { Capital } \\
\text { social } \\
\text { entrepreneurial }\end{array}$} & CS1 & $\begin{array}{l}\text { Taille du réseau social = nombre de liens (forts et faibles) dans } \\
\text { le réseau entrepreneurial }\end{array}$ \\
\hline & CS2 & Nombre de liens faibles \\
\hline & $\mathrm{CS} 3$ & $\begin{array}{l}\text { DS = diversité structurale = hétérogénéité des alters dans le } \\
\text { réseau social entrepreneurial }\end{array}$ \\
\hline \multirow{3}{*}{$\begin{array}{l}\text { Accès aux } \\
\text { ressources } \\
\text { financières } \\
\text { octroyées par } \\
\text { les capitalistes } \\
\text { de risque }\end{array}$} & AF1 & $\begin{array}{l}\text { Le nombre d'articles qui protègent les intérêts du capitaliste de } \\
\text { risque et ses droits de propriété dans la convention que nous } \\
\text { avons signée sont plutôt faibles }\end{array}$ \\
\hline & AF2 & $\begin{array}{l}\text { La période séparant la date de décision d'investissement dans } \\
\text { mon projet et celle de la } 1^{\mathrm{e}} \text { injection effective d'investissement } \\
\text { est courte }\end{array}$ \\
\hline & AF3 & $\begin{array}{l}\text { La différence entre le montant initial de l'investissement } \\
\text { sollicité et celui que j'ai réellement obtenu est négligeable }\end{array}$ \\
\hline \multirow{4}{*}{$\begin{array}{l}\text { Accès aux } \\
\text { informations } \\
\text { utiles }\end{array}$} & AI1 & $\begin{array}{l}\text { Je peux facilement rechercher et localiser les informations } \\
\text { technico-commerciales requises }\end{array}$ \\
\hline & $\mathrm{AI} 2$ & $\begin{array}{l}\text { Il m'est relativement facile d'accéder aux informations } \\
\text { technico-commerciales requises }\end{array}$ \\
\hline & $\mathrm{AI} 3$ & $\begin{array}{l}\text { J'arrive à obtenir les informations technico-commerciales dont } \\
\text { j'aurais besoin facilement }\end{array}$ \\
\hline & AI4 & $\begin{array}{l}\text { Je peux rapidement accéder aux informations technico- } \\
\text { commerciales sollicitées }\end{array}$ \\
\hline
\end{tabular}




\section{RÉFÉRENCES}

Adler, P.S. et Kwon, S.W. (2002). Social capital : prospects for a new concept. Academy of Management Review, 27(1), 17-40.

Aldrich, H.E. et Ruef, R. (2006). Organizations Evolving (2 édition). Londres, Sage Publications.

Anderson, A.R. et JACK, S. (2002). The articulation of social capital in entrepreneurial networks : a glue or a lubricant? Entrepreneurship and Regional Development, 14(3), 193-210.

Anderson, A., PArk, J. et Jack, S. (2007). Entrepreneurial social capital. Conceptualizing social capital in new high tech firms. International Small Business Journal, 25(3), 245-272.

BAR-On, R. (2005). The Bar-On model of emotional-social intelligence. Special issue on emotional intelligence. Psicothema, 18(supplément), 1-6.

BARON, A.R. (2004a). The potential role of entrepreneurs' social skills in the success of new ventures : why the ability to get along with others really matters. Dans W.G. Carter, K.G. Shaver, N.M. Carter et P.D. Reynolds (dir.), Handbook of entrepreneurial dynamics : the process of business creation (p. 220233). Newbury Park, Californie, Sage Publications.

Baron, A.R. (2004b). Social skills. Dans W.B Gartner, N.M. Shaver et P.D. Carter, Handbook of entrepreneurial dynamics : the process of business creation (p. 220-233). Thousand Oaks, Sage Publications.

BARON, A.R. et Brush, C. (1999). The role of social skills in entrepreneur's success. Récupéré le 4 avril 2009 du site : http://www.babson.edu.

Baron, A.R. et Kenny, D.A. (1986). The moderator-mediator variable distinction in social psychological research : conceptual, strategic, and statistical considerations. Journal of Personality and Social Psychology, 51(6), 1173-1182.

Baron, A.R. et Markman, G.D. (2000). Beyond social capital : how social skills can enhance entrepreneurs' success. Academy of Management Executive, 14(1), 106-116.

Baron, A.R. et Markman, G.D. (2003). Beyond social capital : the role of entrepreneurs' social competence in their financial success. Journal of Business Venturing, 18(1), 41-60.

BARON, A.R. et TANG, J. (2009, avril). Entrepreneurs' social skills and new venture performance : mediating mechanisms and cultural generality. Journal of Management, 35(2), 282-306.

Batjargal, B. et Liu, M. (2004). Entrepreneurs' access to private equity in China : the role of social capital. Organization Science, 15(2), 159-200.

Besson, D. et Haddadj, J.S. (2003, juin). Adaptation internationale des échelles de mesure entre universalisme et culturalisme : application à la mesure de l'environnement de l'entreprise. Dans les Actes de la $12^{\mathrm{e}}$ Conférence de l'Association internationale de management et stratégie. Carthage/Tunis, Tunisie. Récupéré le 8 janvier 2009 sur le site : http://www.strategie-aims.com/events/conferences/11xiieme-conference-de-l-aims.

Bhagavatula, S., Elfring, T., Van Tilburg, A. et van de Bunt, G. (2010). How social and human capital influence opportunity recognition and resource mobilization in india's handloom industry? Journal of Business Venturing, 25(3), 245-260.

Bolino, M.C. et Turnley, W.H. (1999). Measuring impression management in organizations : a scale development based on the Jones and Pittman taxonomy. Organizational Research Methods, 2(2), 187206. 
Borgatti, S.P., Everett, M.G. et Freeman, L.C. (2002). Software for social networks analysis. Récupéré le 2 mai 2011 sur le site : www.analytictech.com.

Bruyat, C. (1993). Création d'entreprise : contributions épistémologiques et modélisation (thèse de doctorat en sciences de gestion). Université Pierre Mendès, École supérieure des affaires, Grenoble II.

Bruyat, C. et Julien, P.-A. (2001). Defining the field of research entrepreneurship. Journal of Business Venturing, 16(2), 165-180.

BuRT, R. (1992). Structural holes, the social structure of competition. Cambridge, Harvard University Press.

BURT, R. (1997). A note on social capital and network content. Social Networks, 19(4), 355-373.

BurT, R. (2000). The network structure of social capital. Dans R.I. Sutton et B.M. Staw (dir.), Research in Organizational Behavior (p. 345-423). Greenwich, Connecticut, JAI Press.

BURT, R. (2010). Neighbor networks : competitive advantage local and personal. Oxford, Oxford University Press.

Caceres, R.C. et Vanhamme, J. (2003). Les process modérateurs et médiateurs : distinction conceptuelle, aspects analytiques et illustrations. Recherche et applications en marketing, 18(2), 67-100.

Cantner, U. et Stuetzer, M. (2010). The use and affect of social capital in new venture creation-solo entrepreneurs versus new venture teams. Jena Economic Research Papers (n²010). Récupéré le 11 mars 2009 du site : www.jenecon.de.

Carter, N.M., Williams, M. et Reynolds, P.D. (1997). Discontinuance among new firms in retail : the influence of initial resources, strategy, and gender. Journal of business venturing, 12(2), 125-145.

Cavazotte, F., Moreno, V. et Hickmann, M. (2012). Effects of leader intelligence, personality and emotional intelligence on transformational leadership and managerial performance. The Leadership Quarterly, 23(3), 443-455.

Chabaud, D. et Nigigol, J. (2010). Quels réseaux sociaux dans la formation de l'opportunité d'affaires. Revue française de gestion, 7(206), 129-147.

Chandler, G.N. et Hanks, S.H. (1994). Founder competence, the environment, and venture performance. Waco, Texas, ETP/Baylor University.

Chi, L., Fang, R., Chen, M. et Baron, R.A. (2008, juin). Bringing social skills into social networks. Conférence au ICS. Halifax, Nouvelle-Écosse, Canada. Récupéré le 12 octobre 2011 sur le site : www. babson.edu.

CHIN, W.W. (1998a). Issues and opinion on structural equation modeling. MIS Quarterly, 22(1), 7-16.

CHIn, W.W. (1998b). The partial least squares approach to structural equation modeling. Dans G.A. Marcoulides (dir.), Modern methods for business research (p. 295-358). Mahwah, New Jersey, Lawrence Erlbaum.

Chin, W.W., Marcolin, B.L. et. Newsted, P.R. (1996). A partial least squares latent variable modeling approach for measuring interaction effects: results from a Monte Carlo simulation study and voice mail emotion/adoption study (p. 21-41). Proceedings of the seventeenth international conference on information systems. Cleveland, Ohio. Récupéré le 18 juillet 2012 sur le site : http://disc-nt.cba.uh.edu/ chin/icis96.pdf.

Chin, W.W., Marcolin, B.L. et Newsted, P.R. (2003). A partial least squares latent variable modeling approach for measuring. International Information Systems Research, 14(2), 189-217. 
Cialdini, R.B. (2000). Influence : science and practice (4 édition). Boston, Allyn \& Bacon.

Coleman, J.S. (1990). Foundations of social theory. Cambridge, Massachusetts, Harvard University Press.

Cooper, A.C., Folta, T. et Woo, C.Y. (1991). Information acquisition and performance by start-up firms. Dans N.C. Churchill, W.D. Bygrave, J.C. Covin, D.L. Sexton, D.P. Slevin, K.H. Vesper et W.E. Wetzel (dir.), Frontiers of entrepreneurship research (p. 276-290). Babson Park, Massachusetts, Babson College. Récupéré le 18 juillet 2012 sur le site : http://fusionmx.babson.edu/entrep/fer/.

Cronbach, L.J. (1951). Coefficient alpha and the internal structure of tests. Psychometrika, 16(3), 297334.

Davidsson, P. et Honig, B. (2003). The role of social and human capital among nascent entrepreneurs. Journal of Business Venturing, 18(3), 301-331.

DeFFAIns-Crapsky, C. (2011). Le rôle des business angels dans le financement de l'innovation radicale (document de travail du GRANEM n²011-02-027, UMR-RA, n49). Université d'Angers, France.

Depaulo, B.M. (1994). Spotting lies : can human learn to do better ? Current Direction in Psychological Science, 3(3), 83-86.

Diener, E. et Seligman, M.E.P. (2002). Very happy people. Psychological Science, 13(1), 80-83.

Dillard, J.P. (2004). The goals-plans-action model of interpersonal influence. Dans J.S. Seiter et R.H. Gass (dir.), Perspectives on persuasion, social influence, and compliance Gaining (p. 185-206). Boston/ New York/San Francisco, Pearson Education.

Dillard, J.P. et Marshal, L.J. (2003). Persuasion as a social skill. Dans J.O. Greene et B.R. Burleson (dir.), Handbook of communication and social interaction skills (p. 479-514). Londres, Lawrence Erlbaum Associates Publishers.

Dubini, P. et Aldrich, H. (1991). Personal and extended networks are central to the entrepreneurial process. Journal of Business Venturing, 6(5), 305-313.

Elfring, T. et Hulsink, W. (2003). Networks in entrepreneurship : the case of high technology firms. Small Business Economics, 21(4), 409-422.

FAyolle, A. (2005). Introduction à l'entrepreneuriat. Paris, Dunod.

Fayolle, A. (2007). Entrepreneurship and new venture creation: the dynamic of the entrepreneurial process. Cambridge, Cambridge University Press.

Ferrary, M. et Granovetter, M. (2009). The role of venture capital firms in Silicon Valley's complex innovation network. Economy and Society, 38(2), 326-359.

Ferris, G.R., Davidson, S.L. et Perrewé, P.L. (2005). Political skill at work: impact on work effectiveness. Mountain View, Californie, Davies-Black.

Ferris, G.R., Treadway, D.C., Kolodinsky, R.W., Hochwarter, W.A. et Kacmar, C.J. (2005). Development and validation of the political skill inventory. Journal of Management, 31(1), 126-152.

Ferris, G.R., Treadway, D.C., Perrewé, P.L., Brouer, R.L., Douglas, C. et Lux, S. (2007). Political skill in organizations. Journal of Management, 33(3), 290-320.

Ferris, G.R., Witt, L.A. et Hochwarter, W.A. (2001). Interaction of social skill and general mental ability on job performance and salary. Journal of Applied Psychology, 86(6), 1075-1082.

Florin, J., Lubatkin, M. et Schulze, W. (2003). A social capital model of high-growth ventures. Academy of Management Journal, 46(3), 374-384. 
Fornell, C. et LARCKer, D.F. (1981). Evaluating structural equation models with unobservable variables and measurement error. Journal of Marketing Research, 18(1), 39-50.

Fornoni, M., Arribas, I. et. Vila, J.E. (2012). An entrepreneur's social capital and performance : the role of access to information in the argentinean case. Journal of Organizational Change Management, 25(5), 682-698.

Gefen, D. et Straub, D. (2005). A practical guide to factorial validity using PLS-Graph : tutorial and annotated example. Communications of the Association for Information Systems. 16(4), 91-109.

Goleman, D.P. (1998). Emotional intelligence that lead to success. New York, Basic Books.

Goleman, D.P. (2001). An EI-based theory on performance. Dans C. Chernis et D. Goleman (dir.), The emotionally intelligent workplace : how to select for, measure, and improve emotional intelligence in individuals, groups, and organizations (p. 27-44). San Francisco, Jossey-Bass.

Goleman, D.P. (2006). Social intelligence. The new science of human relationships. New York, Bantam Books.

Goleman, D.P. et. Boyatzis, R.E. (2008). Social intelligence and the biology of leadership. Harvard Business Review, 86(9), 74-81.

Goleman, D.P, Boyatzis, R.E. et Mckee, A. (2002). Primal leadership : realizing the power of emotional intelligence ( $1^{\mathrm{e}}$ édition). Boston, Harvard Business School Press.

Granovetter, M. (1983). The strength of weak ties : a network theory revisited. Sociological Theory, 1(1), 201-233.

Greene, P.G. et Brown, T.E. (1997). Resource needs and the dynamic capitalism typology. Journal of Business Venturing, 12(3), 161-173.

Greve, A. et Salaff, J.W. (2003). Social networks and entrepreneurship. Entrepreneurship Theory and Practice, 28(4), 1-22.

Groen, A.J. (2005). Knowledge intensive entrepreneurship in networks : towards a multi-level/ multi-dimensional approach. Journal of Enterprising Culture, 13(1), 69-88.

Hansen, M.T. (1999). The search transfer problem : the role of weak ties in sharing knowledge across organizational sub-units. Administrative Science Quaterly, 41(1), 82-111.

Henseler, J., Ringle, C.M. et Sinkovics, R.R. (2009). The use of partial least squares path modeling in international marketing. Advances in international Marketing, 20(1), 277-319.

Hite, J.M. et Hesterly, W.S. (2001).The evolution of firm networks : from emergence to early growth of the firm. Strategic Management Journal, 22(3), 275-286.

Hochwarter, W.A., Treadway, D.C., Witt, L.A. et Ferris, G.R. (2006). The interaction of social skills and organizational support on job performance. Journal of Applied Psychology, 91(2), 482-489.

InkPen, A.C. et Tsang, E.W. (2005). Social capital, networks, and knowledge transfer. Academy of Management Review, 30(1), 146-165.

Jensen, J. et Greve, A. (2002). Does the degree of redundancy in social networks influence the success of business start-ups. International Journal of Entrepreneurial Behavior and Research, 8(5), 254-267.

Julien, P.-A. (2005). Entrepreneuriat régional et économie de la connaissance - une métaphore des romans policiers. Québec, Canada, Presses de l'Université du Québec. 
Kim, P.H. et Aldrich, H.E. (2005, juillet). Social capital and entrepreneurship. Foundations and trends in Entrepreneurship, 1(2), 55-104.

Lazega, E. et Pattison, P. (2001). Social capital as social mechanisms and collective assets : the example of status auctions among colleagues. Dans N. Lin, K.S. Cook, et R.S. Burt (dir.), Social capital : theory and research (p. 185-208). New york, Aldine de Gruyter.

Lee, Y.W., Strong, D.M., Kahn, B.K. et Wang, R.Y. (2002). AIMQ : a methodology for information quality assessment. Information and Management, 40(2), 133-146.

Lee, R., Tüselmann, H., Jayawarna, D. et Rouse, J. (2011). Investigating the social capital and resource acquisition of entrepreneurs residing in deprived areas of England. Environment and planning C: government and policy, 29(6), 1054-1072.

LIN, N. (2001). Social capital : a theory of social structure and action. Cambridge, Cambridge University Press.

Lochner, K., Kawachi, I. et Kennedy, B.P. (1999). Social capital : a guide to its measurement. Health \& Place, 5(4), 259-270.

MARlowe JR., H.A. (1986). Social intelligence : evidence for multidimensionality and construct independence. Journal of Educational Psychology, 78(1), 52-58.

MARKman, G.D. et BARON, R.A. (1998). Social skills and entrepreneur's financial success : evidence that the ability to get along with others really matters. Frontiers of Entrepreneurship research. Babson Park, Massachusetts, Babson College. Récupéré le 3 avril 2010 sur le site : http://fusionmx.babson. edu/entrep/fer/.

Maurer, I. et Ebers, M. (2006). Dynamics of social capital and their performance implications : lessons from biotechnology start-ups. Administrative Science Quaterly, 51(2), 262-292.

Metts, S. et Grohsкopf, E. (2003). Impression management : goals, strategies and skills. Dans J.O. Greene et B.R. Berleson (dir.), Handbook of communication and social interaction skills (p. 357-400). Mahwa, New Jersey, Routledge Communication series.

Mukamurera, J., Lacourse, F. et Couturier, Y. (2006). Des avancées en analyse qualitative : pour une transparence et une systématisation des pratiques. Recherche Qualitative, 26(1), 110-138.

Nahapiet, J. et Ghoshal, S. (1998). Social capital, intellectual capital and the organizational advantage. The Academy of Management Review, 23(2), 242-266.

Neergaard, H., et Madsen, H. (2004). Knowledge intensive entrepreneurship in a social capital perspective. Journal of Enterprising Culture, 12(2), 105-125.

Nunnally, J.C. (1978). Psychometric theory. New York, McGraw-Hill.

Omrane, A. (2013). Rôle des compétences sociales et du capital social et accès aux ressources externes en création d'entreprise (thèse de doctorat en sciences de gestion). Université Jean Moulin de Lyon 3, Lyon.

Omrane, A., Fayolle, A. et Zeribi, O. (2011). Le processus entrepreneurial et les compétences entrepreneuriales : une approche dynamique. La Revue des sciences de gestion, 5(251), 91-100.

Ozgen, E. et BARON, R.A. (2007). Social sources of information in opportunity recognition : effects of mentors, industry networks, and professional forums. Journal of Business Venturing, 22(2), 174-192.

Perrewé, P.L., Ferris, G.R., Stoner, J.S. et Brouer, R.L. (2007). The positive role of political skill in organizations. Dans D.L. Nelson et C.L. Cooper (dir.), Positive organizational behavior : accentuating the positive at work (p. 117-128). Thousand Oaks, Californie, Sage Publications. 
Perrewé, P.L., Zellars, K.L., Ferris, G.R., Rossi, G.R., Kacmar, C.J. et Ralston, D.A. (2004). Neutralizing job stressors : political skill as an antidoteto the dysfunctional consequence of role conflict stressors. Academy of Management Journal, 47(1), 141-152.

Ringle, C.M. (2004). Gütemaße für den partial least squares-ansatz zur bestimmung von kausalmodellen (document de travail $\mathrm{n}^{\circ} 16$ ). Université de Hamburg, Institut d'économie industrielle et d'organisation, Allemagne.

Seibert, B., Kraimer, M.L. et Liden, R.C. (2001). A social capital theory of career success. Academy of Management Journal, 44(2), 219-237.

Shane, S. et CABle, D. (2002). Network ties, reputation and the financing of new ventures. Management science, 48(3), 364-381.

Shane, S. et Delmar, F. (2004). Planning for the market : business planning before marketing and the continuation of organizing efforts. Journal of Business Venturing, 19(6), 767-785.

Shepherd, D.A. et ZaCharakis, A. (2001). The venture capitalist-entrepreneur relationship : control, trust and confidence in co-operative behavior. Venture Capital, 3(2), 129-149.

Stvilia, B., Gasser, L., Twidale, M.B. et. Smith, L. (2007). A framework for information quality assessmen. Journal of American Society for Information Science and Technology, 58(12), 1720-1733.

Talavera, O., Xiong, L. et Xiong, X. (2012). Social capital and access to bank financing : the case of chinese entrepreneurs. Emerging Markets Finance \& Trade, 48(1), 55-69.

Thorndike, E.L. (1920). Intelligence and its use. Harper's Magazine, (140), 227-235.

ToRnikoski, E.T. et ScotT, L.N. (2007). Exploring the determinants of organizational emergence : a legitimacy perspective. Journal of Business Venturing, 22(2), 311-335.

Tsai, W.P. et Ghoshal, S. (1998). Social capital and value creation : the role of intrafirm networks. Academy of Management Journal, 41(4), 464-476.

Urbach, N. et Ahlemann, F. (2010). Structural equation modeling in information systems - research using partial least squares. Journal of Information Technology Theory and Practice, 11(2), 5-40.

UzzI, B. (1997). Social structure and competition in inter-firm networks : the paradox of embeddedness. Administrative Science Quarterly, 42(1), 35-67.

UzzI, B. (1999). Embeddedness in the making of financial capital : how social relations and networks benefit firms seeking financing. American Sociology Review, 64(4), 481-505.

Van Der GaAg, M.P.J. et Snijders, T.A.B. (2005). The resource generator : social capital quantification with concrete items. Social Networks, 27(1), 1-29.

Witt, L.A. et Ferris, G.R. (2003). Social skill as moderator of the conscientiousness-performance relationship : convergent results across four studies. Journal of Applied Psychology, 88(5), 809-820.

Wu, S.Y., Turban, D.B. et Cheung, Y.H. (2012). Social skill in workplace mentoring relationships. Journal of Organizational Culture, Communications and Conflict, 16(2), 61-73.

Yli-Renko, H., Autio, E. et Sapienza, H.J. (2001). Social capital, knowledge acquisition, and knowledge exploitation in young, technology-based firms. Strategic Management Journal, 22(6-7), 587-613.

Zacharakis, A.L., Mcmullen, J.S. et Shepherd, D.A. (2007). Venture capitalists' decision policies across three countries : an institutional theory perspective. Journal of International Business Studies, 38(5), 691-708. 
Zhao, X.Y., Frese, M. et Giardini, A. (2010). Business owners' network size and business growth in China : the role of comprehensive social competency. Babson-Kauffman Entrepreneurship Research Conference. Babson Park, Massachusetts, Babson College. Récupéré le 19 mai 2010 sur le site : http:// bs.bnu.edu.cn/docs/20110929105105861818.pdf.

Zhu, K., Kraemer, K.L., Gurbaxani, V. et Xu, S.X. (2006). Migration to open-standard interorganizational systems : network effects, switching costs, and path dependency. MIS Quarterly, 30(1), 515-539. 